\title{
Construction of class fields over cyclotomic fields
}

\author{
Ja Kyung Koo and Dong Sung Yoon
}

\begin{abstract}
Let $\ell$ and $p$ be odd primes. For a positive integer $\mu$, let $k_{\mu}$ be the ray class field of $k=\mathbb{Q}\left(e^{2 \pi i / \ell}\right)$ modulo $2 p^{\mu}$. We present certain class fields $K_{\mu}$ of $k$ such that $k_{\mu} \subset$ $K_{\mu} \subset k_{\mu+1}$, and we provide a necessary and sufficient condition for $K_{\mu}=k_{\mu+1}$. We also construct, in the sense of Hilbert, primitive generators of the field $K_{\mu}$ over $k_{\mu}$ by using Shimura's reciprocity law and special values of theta constants.
\end{abstract}

\section{Introduction}

In his 12th problem, Hilbert asked what kind of analytic functions and algebraic numbers are necessary to construct all abelian extensions of given number fields. For any number field $K$ and a modulus $\mathfrak{m}$ of $K$, it is well known (see [29] or [7, Theorem 8.6]) that there is a unique maximal abelian extension of $K$ unramified outside $\mathfrak{m}$, which is called the ray class field of $K$ modulo $\mathfrak{m}$. Hence, as a first step toward the problem we need to construct ray class fields for given number fields. Historically, over imaginary quadratic number fields $K$, Hasse [10] constructed the ray class field of $K$ by making use of the Weber function and the elliptic modular function. After Hasse, many people investigated this theme (see, e.g., [2]-[9], [12], [18], [20]-[22], [28]). On the other hand, over any other CM-fields $K$ with $[K: \mathbb{Q}]>2$, not much seems to be known so far. For instance, over a cyclotomic number field $K$ with odd relative class number, Shimura [23] showed that the Hilbert class field of $K$ is generated by that of the maximal real subfield of $K$ and the unramified abelian extensions of $K$ obtained by the fields of moduli of two certain polarized abelian varieties having subfields of $K$ as endomorphism algebras. By making use of Galois representation, Ribet [19] constructed unramified abelian, degree- $p$ extensions of $K=\mathbb{Q}\left(e^{2 \pi i / p}\right)$ for all irregular primes $p$ (see also [17]). Furthermore, Komatsu [14] investigated a certain class field of $K=\mathbb{Q}\left(e^{2 \pi i / 5}\right)$ and constructed its normal basis by means of Siegel modular functions.

Now, let $n$ be a positive integer, let $k$ be a CM-field with $[k: \mathbb{Q}]=2 n$, let $k^{*}$ be its reflex field, and let $z_{0}$ be the associated CM-point (see Section 3). Shimura [27] showed that if $f$ is a Siegel modular function which is finite at $z_{0}$, then the

Kyoto Journal of Mathematics, Vol. 56, No. 4 (2016), 803-829

DOI $10.1215 / 21562261-3664923$, (C) 2016 by Kyoto University

Received August 31, 2015. Revised October 26, 2015. Accepted November 4, 2015.

2010 Mathematics Subject Classification: Primary 11R37, 11F46; Secondary 11G15, 14 H42. 
special value $f\left(z_{0}\right)$ belongs to some abelian extension (i.e., class field) of $k^{*}$. His reciprocity law explains Galois actions on $f\left(z_{0}\right)$ in terms of the action of the group $G_{\mathbb{A}+}$ on $f$ (Proposition 3.2). Here $G_{\mathbb{A}+}=\prod_{p}^{\prime} \operatorname{GSp}_{2 n}\left(\mathbb{Q}_{p}\right) \times \operatorname{GSp}_{2 n}^{+}(\mathbb{R})$ is the restricted product with respect to the subgroups $\operatorname{GSp}_{2 n}\left(\mathbb{Z}_{p}\right)$ of $\operatorname{GSp}_{2 n}\left(\mathbb{Q}_{p}\right)$. Shimura [26] also constructed Siegel modular functions by the quotient of two theta constants

$$
\Phi_{(r, s)}(z)=\frac{\sum_{x \in \mathbb{Z}^{n}} e\left(\frac{1}{2}^{t}(x+r) z(x+r)+{ }^{t}(x+r) s\right)}{\sum_{x \in \mathbb{Z}^{n}} e\left(\frac{1}{2}^{t} x z x\right)},
$$

for $r, s \in \mathbb{Q}^{n}$, and explicitly described the Galois actions on the special values of theta functions (see Section 5).

In this article, we mainly consider the case of cyclotomic number fields $k=\mathbb{Q}\left(e^{2 \pi i / \ell}\right)$ for any odd prime $\ell$. Let $p$ be an odd prime, and let $\mu$ be a positive integer. We denote by $k_{\mu}$ the ray class field of $k$ modulo $2 p^{\mu}$. In Section 4 , we define the class field $K_{\mu}$ of $k$ such that $k_{\mu} \subset K_{\mu} \subset k_{\mu+1}$, which would be an extension of Komatsu's result [14, Proposition 1]. We shall first find the exact degree of $K_{\mu}$ over $k_{\mu}$ for any odd prime $\ell$ (Theorem 4.5). We shall further provide a necessary and sufficient condition for $K_{\mu}$ to be the ray class field $k_{\mu+1}$ (Corollary 4.6). In Section 6, as Hilbert proposed, by using Shimura's reciprocity law we shall construct a primitive generator of $K_{\mu} / k_{\mu}$ in terms of a special value of $\Phi_{(r, s)}(z)$ for some $r, s \in \mathbb{Q}^{n}$ at the CM-point corresponding to the polarized abelian variety of genus $n=(\ell-1) / 2$ (Theorem 6.4).

\section{NOTATION 1.1}

For $z \in \mathbb{C}$, we denote by $\bar{z}$ the complex conjugate of $z$ and by $\operatorname{Im}(z)$ the imaginary part of $z$, and we put $e(z)=e^{2 \pi i z}$. If $R$ is a ring with identity and $r, s \in \mathbb{Z}_{>0}$, then $M_{r \times s}(R)$ indicates the ring of all $r \times s$ matrices with entries in $R$. In particular, we set $M_{r}(R)=M_{r \times r}(R)$. The identity matrix of $M_{r}(R)$ is written as $1_{r}$, and the transpose of a matrix $\alpha$ is denoted by ${ }^{t} \alpha$. Additionally, $R^{\times}$stands for the group of all invertible elements of $R$. When $G$ is a group and $g_{1}, g_{2}, \ldots, g_{r}$ are elements of $G$, let $\left\langle g_{1}, g_{2}, \ldots, g_{r}\right\rangle$ be the subgroup of $G$ generated by $g_{1}, g_{2}, \ldots, g_{r}$, and let $G^{n}$ be the subgroup $\left\{g^{n} \mid g \in G\right\}$ of $G$ for $n \in \mathbb{Z}_{>0}$. Moreover, when $H$ is a subgroup of $G$, let $|G: H|$ be the index of $H$ in $G$. For a finite algebraic extension $K$ over $F,[K: F]$ denotes the degree of $K$ over $F$. We let $\zeta_{N}=e^{2 \pi i / N}$ be a primitive $N$ th root of unity for a positive integer $N$.

\section{Siegel modular forms}

We shall briefly present necessary facts about Siegel modular forms and explain the action of $G_{\mathbb{A}+}$ on the Siegel modular functions whose Fourier coefficients lie in some cyclotomic fields.

Let $n$ be a positive integer, and let $G$ be the algebraic subgroup of $\mathrm{GL}_{2 n}$ defined over $\mathbb{Q}$ such that

$$
G_{\mathbb{Q}}=\left\{\left.\alpha \in \mathrm{GL}_{2 n}(\mathbb{Q})\right|^{t} \alpha J \alpha=\nu(\alpha) J \text { with } \nu(\alpha) \in \mathbb{Q}^{\times}\right\},
$$


where

$$
J=J_{n}=\left[\begin{array}{cc}
0 & -1_{n} \\
1_{n} & 0
\end{array}\right] .
$$

Let $G_{\mathbb{A}}$ be the adelization of $G$, let $G_{0}$ be the non-Archimedean part of $G_{\mathbb{A}}$, and let $G_{\infty}$ be the archimedean part of $G_{\mathbb{A}}$. We extend the multiplier map $\nu: G_{\mathbb{Q}} \rightarrow \mathbb{Q}^{\times}$ to a continuous map of $G_{\mathbb{A}}$ into $\mathbb{Q}_{\mathbb{A}}^{\times}$, which we denote again by $\nu$. Then we put $G_{\infty+}=\left\{x \in G_{\infty} \mid \nu(x) \gg 0\right\}$ and $G_{\mathbb{A}+}=G_{0} G_{\infty+}$. Here $t \gg 0$ means $t_{v}>0$ for all archimedean primes $v$ of $\mathbb{Q}$. For a positive integer $N$, let

$$
\begin{aligned}
R_{N}= & \mathbb{Q}^{\times} \cdot\left\{a \in G_{\mathbb{A}+} \mid a_{q} \in \mathrm{GL}_{2 n}\left(\mathbb{Z}_{q}\right), a_{q} \equiv 1_{2 n} \quad\left(\bmod N \cdot M_{2 n}\left(\mathbb{Z}_{q}\right)\right)\right. \\
& \text { for all primes } q\} \\
\Delta= & \left\{\left[\begin{array}{cc}
1_{n} & 0 \\
0 & x \cdot 1_{n}
\end{array}\right] \mid x \in \prod_{q} \mathbb{Z}_{q}^{\times}\right\} \\
G_{\mathbb{Q}+}= & \left\{\alpha \in G_{\mathbb{Q}} \mid \nu(\alpha)>0\right\} .
\end{aligned}
$$

\section{PROPOSITION 2.1}

For every positive integer $N$, we have

$$
G_{\mathbb{A}+}=R_{N} \Delta G_{\mathbb{Q}+} \cdot
$$

Proof

See [24, Proposition 3.4] and [25, p. 535, (3.10.3)].

Let $\mathbb{H}_{n}=\left\{z \in M_{n}(\mathbb{C}) \mid{ }^{t} z=z, \operatorname{Im}(z)>0\right\}$ be the Siegel upper half-space of degree $n$. Here, for a Hermitian matrix $\xi$ we write $\xi>0$ to mean that $\xi$ is positive definite. We define the action of an element $\alpha=\left[\begin{array}{ll}A & B \\ C & D\end{array}\right]$ of $G_{\mathbb{Q}+}$ on $\mathbb{H}_{n}$ by

$$
\alpha(z)=(A z+B)(C z+D)^{-1},
$$

where $A, B, C, D \in M_{n}(\mathbb{Q})$. For every positive integer $N$, let

$$
\Gamma(N)=\left\{\gamma \in \operatorname{Sp}_{2 n}(\mathbb{Z}) \mid \gamma \equiv 1_{2 n} \quad\left(\bmod N \cdot M_{2 n}(\mathbb{Z})\right)\right\}
$$

For an integer $m$, a holomorphic function $f: \mathbb{H}_{n} \rightarrow \mathbb{C}$ is called a (classical) Siegel modular form of weight $m$ and level $N$ if

$$
f(\gamma(z))=\operatorname{det}(C z+D)^{m} f(z)
$$

for all $\gamma=\left[\begin{array}{cc}A & B \\ C & D\end{array}\right] \in \Gamma(N)$ and $z \in \mathbb{H}_{n}$, plus the requirement when $n=1$ that $f$ is holomorphic at every cusp. In particular, $f(z)$ has a Fourier expansion of the form

$$
f(z)=\sum_{\xi} A(\xi) e(\operatorname{tr}(\xi z) / N)
$$

with $A(\xi) \in \mathbb{C}$, where $\xi$ runs over all positive semidefinite half-integral symmetric matrices of degree $n$ (see [13, Section 4, Theorem 1]). Here, a symmetric matrix 
$\xi \in M_{n}(\mathbb{Q})$ is called half-integral if $2 \xi$ is an integral matrix whose diagonal entries are even.

For a subring $R$ of $\mathbb{C}$, let $\mathcal{M}_{m}(\Gamma(N), R)$ be the vector space of all Siegel modular forms $f$ of weight $m$ and level $N$ whose Fourier coefficients $A(\xi)$ belong to $R$, and let $\mathcal{M}_{m}(R)=\bigcup_{N=1}^{\infty} \mathcal{M}_{m}(\Gamma(N), R)$. We denote by $\mathcal{A}_{m}(R)$ the set of all meromorphic functions of the form $g / h$ with $g \in \mathcal{M}_{r+m}(R), 0 \neq h \in \mathcal{M}_{r}(R)$ (with any $r \in \mathbb{Z})$, and we denote by $\mathcal{A}_{m}(\Gamma(N), R)$ the set of all $f \in \mathcal{A}_{m}(R)$ satisfying (2.1). In particular, we set

$$
\begin{aligned}
\mathcal{F}_{N} & =\mathcal{A}_{0}\left(\Gamma(N), \mathbb{Q}\left(\zeta_{N}\right)\right), \\
\mathcal{F} & =\bigcup_{N=1}^{\infty} \mathcal{F}_{N} .
\end{aligned}
$$

For every algebraic number field $K$, let $K_{a b}$ be the maximal abelian extension of $K$, and let $K_{\mathbb{A}}^{\times}$be the idèle group of $K$. By class field theory, every element $x$ of $K_{\mathbb{A}}^{\times}$acts on $K_{a b}$ as an automorphism. We then denote this automorphism by $[x, K]$. On the other hand, every element of $G_{\mathbb{A}+}$ acts on $\mathcal{F}$ as an automorphism (see [26, p. 680]). If $x \in G_{\mathbb{A}+}$ and $f \in \mathcal{F}$, then we denote by $f^{x}$ the image of $f$ under $x$.

PROPOSITION 2.2

Let $f(z)=\sum_{\xi} A(\xi) e(\operatorname{tr}(\xi z) / N) \in \mathcal{F}_{N}$. Then we get the following.

(i) $f^{\beta}=f$ for $\beta \in R_{N}$. Moreover, $\mathcal{F}_{N}$ is the subfield of $\mathcal{F}$ consisting of all the $R_{N}$-invariant elements.

(ii) Let $y=\left[\begin{array}{cc}1_{n} & 0 \\ 0 & x \cdot 1_{n}\end{array}\right] \in \Delta$, and let $t$ be a positive integer such that $t \equiv x_{q}$ $\left(\bmod N \mathbb{Z}_{q}\right)$ for all primes $q$. Then we derive

$$
f^{y}=\sum_{\xi} A(\xi)^{\sigma} e(\operatorname{tr}(\xi z) / N)
$$

where $\sigma$ is the automorphism of $\mathbb{Q}\left(\zeta_{N}\right)$ such that $\zeta_{N}^{\sigma}=\zeta_{N}^{t}$.

(iii) $f^{\alpha}=f \circ \alpha$ for $\alpha \in G_{\mathbb{Q}+}$.

Proof

See [26, p. 681] and [27, Theorem 26.8].

\section{Shimura's reciprocity law}

We begin with fundamental but necessary facts about Shimura's reciprocity law from [27, Section 26]. Let $n$ be a positive integer, let $K$ be a CM-field with $[K$ : $\mathbb{Q}]=2 n$, and let $\mathcal{O}_{K}$ be a ring of integers of $K$. Let $\varphi_{1}, \varphi_{2}, \ldots, \varphi_{n}$ be $n$ distinct embeddings of $K$ into $\mathbb{C}$ such that there are no two embeddings among them which are complex conjugates of each other on $K$. Then $\left(K ;\left\{\varphi_{1}, \varphi_{2}, \ldots, \varphi_{n}\right\}\right)$ is a CM-type, and we can take an element $\rho$ in $K$ such that

(i) $\rho$ is purely imaginary, 
(ii) $\operatorname{Im}\left(\rho^{\varphi_{i}}\right)>0$ for all $i=1, \ldots, n$,

(iii) $\operatorname{Tr}_{K / \mathbb{Q}}(\rho \xi) \in \mathbb{Z}$ for all $\xi \in \mathcal{O}_{K}$.

We denote by $v(x)$, for $x \in K$, the vector of $\mathbb{C}^{n}$ whose components are $x^{\varphi_{1}}, \ldots$, $x^{\varphi_{n}}$. The set $L=\left\{v(x) \mid x \in \mathcal{O}_{K}\right\}$ is a lattice in $\mathbb{C}^{n}$. We define an $\mathbb{R}$-bilinear form $E(z, w)$ on $\mathbb{C}^{n}$ by

$$
E(z, w)=\sum_{i=1}^{n} \rho^{\varphi_{i}}\left(z_{i} \overline{w_{i}}-\overline{z_{i}} w_{i}\right) \quad \text { for } z=\left[\begin{array}{c}
z_{1} \\
\vdots \\
z_{n}
\end{array}\right] \text { and } w=\left[\begin{array}{c}
w_{1} \\
\vdots \\
w_{n}
\end{array}\right] .
$$

Then $E$ becomes a nondegenerate Riemann form on the complex torus $\mathbb{C}^{n} / L$ satisfying

$$
E(v(x), v(y))=\operatorname{Tr}_{k / \mathbb{Q}}(\rho x \bar{y}) \quad \text { for } x, y \in K,
$$

which makes it a polarized abelian variety (see [27, pp. 43-44]). Hence, we can find a positive integer $\delta$, a diagonal matrix $\epsilon$ with integral elements, and a complex $n \times 2 n$ matrix $\Omega$ such that (see [27, Lemma 27.2] or [26, p. 675])

(i) $E(\Omega x, \Omega y)=\delta \cdot{ }^{t} x J y$ for $x, y \in \mathbb{R}^{2 n}$,

(ii) $L=\left\{\Omega\left[\begin{array}{l}a \\ b\end{array}\right] \mid a \in \mathbb{Z}^{n}, b \in \epsilon \mathbb{Z}^{n}\right\}$,

(iii)

$$
\epsilon=\left[\begin{array}{cccc}
\epsilon_{1} & & & \\
& \epsilon_{2} & & \\
& & \ddots & \\
& & & \epsilon_{n}
\end{array}\right], \quad \epsilon_{1}=1, \epsilon_{i} \mid \epsilon_{i+1}, \text { for } i=1, \ldots, n-1 .
$$

Now, we write $\Omega=\left[\Omega_{1} \Omega_{2}\right]=\left[v\left(e_{1}\right) v\left(e_{2}\right) \cdots v\left(e_{2 n}\right)\right]$ with $\Omega_{1}, \Omega_{2} \in M_{n}(\mathbb{C})$ and $e_{1}, e_{2}, \ldots, e_{2 n} \in K$, and we put $z_{0}=\Omega_{2}^{-1} \Omega_{1}$. It is well known that $z_{0} \in \mathbb{H}_{n}$. Let $\Phi: K \rightarrow M_{n}(\mathbb{C})$ be a ring monomorphism such that

$$
\Phi(x)=\left[\begin{array}{cccc}
x^{\varphi_{1}} & & & \\
& x^{\varphi_{2}} & & \\
& & \ddots & \\
& & & x^{\varphi_{n}}
\end{array}\right] \quad \text { for } x \in K .
$$

Then we can define a ring monomorphism $h: K \rightarrow M_{2 n}(\mathbb{Q})$ by

$$
\Phi(x) \Omega=\Omega \cdot{ }^{t} h(x) \quad \text { for } x \in K .
$$

Here, $h(x)=\left[a_{i j}\right]_{1 \leq i, j \leq 2 n}$ is, in fact, the regular representation of $x$ with respect to $\left\{e_{1}, e_{2}, \ldots, e_{2 n}\right\}$, namely, $x e_{i}=\sum_{j=1}^{2 n} a_{i j} e_{j}$. If $\epsilon=1_{n}$, then $L=v\left(\mathcal{O}_{K}\right)=\Omega$. $\mathbb{Z}^{2 n}=\mathbb{Z} v\left(e_{1}\right)+\cdots+\mathbb{Z} v\left(e_{2 n}\right)$ so that $h(x) \in M_{2 n}(\mathbb{Z})$ for $x \in \mathcal{O}_{K}$. One can then readily show that

$$
h(\bar{x})=J^{t} h(x) J^{-1} \quad \text { for } x \in K,
$$

and that $z_{0}$ is the CM-point of $\mathbb{H}_{n}$ induced from $h$ which corresponds to the principally polarized abelian variety $\left(\mathbb{C}^{n} / L, E\right)$ (see [26, pp. 684-685] or [27, 
Section 24.10]). In particular, if we set $S=\left\{x \in K^{\times} \mid x \bar{x} \in \mathbb{Q}^{\times}\right\}$, then $h(S)=$ $\{h(s) \mid s \in S\}=\left\{\alpha \in G_{\mathbb{Q}+} \mid \alpha\left(z_{0}\right)=z_{0}\right\}$.

Let $K^{*}$ be the reflex field of $K$, let $K^{\prime}$ be a Galois extension of $K$ over $\mathbb{Q}$, and extend $\varphi_{i}(i=1, \ldots, n)$ to an element of $\operatorname{Gal}\left(K^{\prime} / \mathbb{Q}\right)$, which we denote again by $\varphi_{i}$. Let $\left\{\psi_{j}\right\}_{j=1}^{m}$ be the set of all embeddings of $K^{*}$ into $\mathbb{C}$ obtained from $\left\{\varphi_{i}^{-1}\right\}_{i=1}^{n}$.

\section{PROPOSITION 3.1}

Let $K, K^{*}$, and $\left\{\psi_{j}\right\}$ be as above.

(i) $\left(K^{*} ;\left\{\psi_{1}, \ldots, \psi_{m}\right\}\right)$ is a primitive CM-type and we have

$$
K^{*}=\mathbb{Q}\left(\sum_{i=1}^{n} x^{\varphi_{i}} \mid x \in K\right) .
$$

(ii) If $b=\prod_{j} a^{\psi_{j}}$ with $a \in K^{*}$, then $b \in K$ and $b \bar{b}=N_{K^{*} / \mathbb{Q}}(a)$.

Proof

See [27, pp. 62-63].

We call the CM-type $\left(K^{*} ;\left\{\psi_{j}\right\}\right)$ the reflex of $\left(K ;\left\{\varphi_{i}\right\}\right)$. By Proposition 3.1, we can define a homomorphism $\varphi^{*}:\left(K^{*}\right)^{\times} \rightarrow K^{\times}$by

$$
\varphi^{*}(a)=\prod_{j=1}^{m} a^{\psi_{j}} \quad \text { for } a \in\left(K^{*}\right)^{\times},
$$

and we have $\varphi^{*}(a) \cdot \overline{\varphi^{*}(a)}=N_{K^{*} / \mathbb{Q}}(a)$ for $a \in\left(K^{*}\right)^{\times}$. The map $h$ can be extended naturally to a homomorphism $K_{\mathbb{A}} \rightarrow M_{2 n}\left(\mathbb{Q}_{\mathbb{A}}\right)$, which we also denote by $h$. Then for every $b \in\left(K^{*}\right)_{\mathbb{A}}^{\times}$we get $\nu\left(h\left(\varphi^{*}(b)\right)\right)=N_{K^{*} / \mathbb{Q}}(b)$ and $h\left(\varphi^{*}(b)^{-1}\right) \in G_{\mathbb{A}+}($ see $[27$, p. 172]).

\section{PROPOSITION 3.2 (SHIMURA'S RECIPROCITY LAW)}

Let $K, h, z_{0}$, and $K^{*}$ be as above. Then for every $f \in \mathcal{F}$ which is finite at $z_{0}$, the value $f\left(z_{0}\right)$ belongs to $K_{a b}^{*}$. Moreover, if $b \in\left(K^{*}\right)_{\mathbb{A}}^{\times}$, then $f^{h\left(\varphi^{*}(b)^{-1}\right)}$ is finite at $z_{0}$ and

$$
f\left(z_{0}\right)^{\left[b, K^{*}\right]}=f^{h\left(\varphi^{*}(b)^{-1}\right)}\left(z_{0}\right)
$$

\section{Proof}

See [27, Theorem 26.8].

\section{REMARK 3.3}

For any $f \in \mathcal{F}$ which is finite at $z_{0}$, the value $f\left(z_{0}\right)$ in fact belongs to the class field $\widetilde{K_{a b}^{*}}$ of $K^{*}$ corresponding to the kernel of $\varphi^{*}$. 


\section{Class fields over cyclotomic fields}

Let $\ell$ and $p$ be odd prime numbers. We also write for simplicity $\zeta=\zeta_{\ell}$. Set $k=\mathbb{Q}(\zeta)$ and $n=(\ell-1) / 2$ so that $2 n=[k: \mathbb{Q}]$. For $1 \leq i \leq 2 n$ we denote by $\varphi_{i}$ the element of $\operatorname{Gal}(k / \mathbb{Q})$ defined by $\zeta^{\varphi_{i}}=\zeta^{i}$. Then $\left(k ;\left\{\varphi_{1}, \varphi_{2}, \ldots, \varphi_{n}\right\}\right)$ is a primitive CM-type and $\left(k ;\left\{\varphi_{1}^{-1}, \varphi_{2}^{-1}, \ldots, \varphi_{n}^{-1}\right\}\right)$ is its reflex (see [27, p. 64]). For a positive integer $\mu$, put

$$
\begin{aligned}
& S_{\mu}=\left\{a \in k^{\times} \mid a \equiv 1 \quad\left(\bmod 2 p^{\mu}\right)\right\}, \\
& \widetilde{S_{\mu}}=\left\{(a) \mid a \in S_{\mu}\right\},
\end{aligned}
$$

where $(a)$ is the principal ideal of $k$ generated by $a$. Let $E$ be the unit group of $k$, and let $k_{\mu}$ be the ray class field of $k$ modulo $2 p^{\mu}$. Then we have

$$
\operatorname{Gal}\left(k_{\mu+1} / k_{\mu}\right) \cong \widetilde{S_{\mu}} / \widetilde{S_{\mu+1}} \cong S_{\mu} E / S_{\mu+1} E \cong S_{\mu} / S_{\mu+1}\left(S_{\mu} \cap E\right)
$$

by class field theory. Further, we set $H_{\mu}=S_{\mu+1}\left(S_{\mu} \cap E\right)$ and

$$
\omega_{\mu, i}= \begin{cases}1+2 p^{\mu} \zeta^{i} & \text { for } 1 \leq i \leq n+1 \\ 1+2 p^{\mu}\left(\zeta^{n}+\zeta^{n+1}-\zeta^{i}-\zeta^{-i}\right) & \text { for } n+2 \leq i \leq 2 n\end{cases}
$$

Since the ring of integers $\mathcal{O}_{k}$ of $k$ is equal to $\mathbb{Z}[\zeta]$ and $S_{\mu} / S_{\mu+1}$ is isomorphic to $\mathcal{O}_{k} / p \mathcal{O}_{k}$ by a mapping

$$
\begin{aligned}
S_{\mu} / S_{\mu+1} & \longrightarrow \mathcal{O}_{k} / p \mathcal{O}_{k}, \\
\left(1+2 p^{\mu} \omega\right) S_{\mu+1} & \longmapsto \omega+p \mathcal{O}_{k} \quad \text { for } \omega \in \mathcal{O}_{k},
\end{aligned}
$$

we obtain $S_{\mu} / S_{\mu+1} \cong(\mathbb{Z} / p \mathbb{Z})^{2 n}$ and

$$
S_{\mu} / S_{\mu+1}=\left\langle\left(1+2 p^{\mu} \zeta\right) S_{\mu},\left(1+2 p^{\mu} \zeta^{2}\right) S_{\mu}, \ldots,\left(1+2 p^{\mu} \zeta^{2 n}\right) S_{\mu}\right\rangle .
$$

Let $B=\left[b_{i j}\right] \in M_{2 n}(\mathbb{Z})$ where $b_{i j}$ is an integer such that $\omega_{\mu, i}=1+$ $2 p^{\mu}\left(\sum_{j=1}^{2 n} b_{i j} \zeta^{j}\right)$. Then we get

$$
B=\left[\begin{array}{cccc|cccc}
1 & & & & 0 & 0 & \cdots & 0 \\
& 1 & & & \vdots & \vdots & & \vdots \\
& & \ddots & & \vdots & \vdots & & \vdots \\
& & & 1 & 0 & 0 & \cdots & 0 \\
\hline & & & 0 & 1 & & & \\
& & -1 & 1 & 1 & -1 & & \\
& . & & \vdots & \vdots & & \ddots & \\
-1 & & & 1 & 1 & & & -1
\end{array}\right] .
$$

Hence, $\quad S_{\mu} / S_{\mu+1}=\left\langle\omega_{\mu, 1} S_{\mu+1}, \omega_{\mu, 2} S_{\mu+1}, \ldots, \omega_{\mu, 2 n} S_{\mu+1}\right\rangle$ because $\operatorname{det}(B)=$ $(-1)^{n-1}$ is prime to $p$. This shows that $S_{\mu} / H_{\mu}=\left\langle\omega_{\mu, 1} H_{\mu}, \omega_{\mu, 2} H_{\mu}, \ldots, \omega_{\mu, 2 n} H_{\mu}\right\rangle$ due to the fact that $H_{\mu} \supset S_{\mu+1}$.

Now, we define an endomorphism $\varphi^{*}$ of $k^{\times}$by

$$
\varphi^{*}(a)=\prod_{i=1}^{n} a^{\varphi_{i}^{-1}} \quad \text { for } a \in k^{\times}
$$


and an endomorphism $\varphi^{+}$of $k$ by

$$
\varphi^{+}(a)=\sum_{i=1}^{n} a^{\varphi_{i}^{-1}} \quad \text { for } a \in k .
$$

We let

$$
\eta_{\mu, i}= \begin{cases}1+2 p^{\mu} \varphi^{+}\left(\zeta^{i}\right) & \text { for } 1 \leq i \leq n+1, \\ 1 & \text { for } n+2 \leq i \leq 2 n,\end{cases}
$$

so that $\varphi^{*}\left(\omega_{\mu, i}\right) H_{\mu}=\eta_{\mu, i} H_{\mu}$ for all $1 \leq i \leq 2 n$. Since $\varphi^{*}\left(H_{\mu}\right) \subset H_{\mu}$, we can define an endomorphism $\widetilde{\varphi_{\mu}^{*}}$ of $S_{\mu} / H_{\mu}$ by $\widetilde{\varphi_{\mu}^{*}}\left(a H_{\mu}\right)=\varphi^{*}(a) H_{\mu}$. Let $K_{\mu}$ be the class field of $k$ corresponding to the kernel of $\widetilde{\varphi_{\mu}^{*}}$. Note that $K_{\mu}=k_{\mu}\left(k_{\mu+1} \cap \widetilde{k_{a b}}\right)$, where $\widetilde{k_{a b}}$ is the class field of $k$ in Remark 3.3. Then we get

$$
\begin{aligned}
\operatorname{Gal}\left(K_{\mu} / k_{\mu}\right) & \cong\left(S_{\mu} / H_{\mu}\right) / \operatorname{ker}\left(\widetilde{\varphi_{\mu}^{*}}\right) \cong \widetilde{\varphi_{\mu}^{*}}\left(S_{\mu} / H_{\mu}\right) \\
& =\left\langle\eta_{\mu, 1} H_{\mu}, \eta_{\mu, 2} H_{\mu}, \ldots, \eta_{\mu, n+1} H_{\mu}\right\rangle .
\end{aligned}
$$

Observe that $K_{\mu}$ is the fixed field of $\left\{\left(\frac{k_{\mu+1} / k}{(\omega)}\right) \mid \omega H_{\mu} \in \operatorname{ker}\left(\widetilde{\varphi_{\mu}^{*}}\right)\right\}$ and

$$
\operatorname{Gal}\left(K_{\mu} / k_{\mu}\right)=\left\langle\left(\frac{K_{\mu} / k}{\left(\omega_{\mu, 1}\right)}\right),\left(\frac{K_{\mu} / k}{\left(\omega_{\mu, 2}\right)}\right), \ldots,\left(\frac{K_{\mu} / k}{\left(\omega_{\mu, n+1}\right)}\right)\right\rangle .
$$

Here, $\left(\frac{k_{\mu+1} / k}{\cdot}\right)$ is the Artin map of $k_{\mu+1} / k$.

\section{PROPOSITION 4.1}

Let $N$ be a positive integer, let $K=\mathbb{Q}\left(\zeta_{N}\right)$, and let $K^{+}$be its maximal real subfield. Let $E$ (resp., $E^{+}$) be the unit group of $K$ (resp., $\left.K^{+}\right)$, and let $W$ be the group of roots of unity in $K$. Then we have

$$
\left|E: W E^{+}\right|= \begin{cases}1 & \text { if } N \text { is a prime power } \\ 2 & \text { if } N \text { is not a prime power. }\end{cases}
$$

Proof

See [30, Corollary 4.13].

\section{PROPOSITION 4.2}

Let $\ell$ be any prime, and let $m \in \mathbb{Z}_{>0}$. Let $\mathbb{Q}\left(\zeta_{\ell^{m}}\right)^{+}$be the maximal real subfield of $\mathbb{Q}\left(\zeta_{\ell^{m}}\right)$, and let $E_{\ell^{m}}^{+}$be its unit group. Further, we let $C_{\ell^{m}}^{+}$be the subgroup of $E_{\ell^{m}}^{+}$generated by -1 and the real units

$$
\xi_{a}=\zeta_{2 \ell^{m}}^{1-a} \cdot \frac{1-\zeta_{\ell^{m}}^{a}}{1-\zeta_{\ell^{m}}} \in \mathbb{R}, \quad 1<a<\frac{\ell^{m}}{2}, \quad \operatorname{gcd}(a, \ell)=1 .
$$

Then

$$
h_{\ell^{m}}^{+}=\left|E_{\ell^{m}}^{+}: C_{\ell^{m}}^{+}\right|
$$

where $h_{\ell^{m}}^{+}$is the class number of $\mathbb{Q}\left(\zeta_{\ell^{m}}\right)^{+}$. 
Proof

See [30, Lemma 8.1 and Theorem 8.2].

\section{LEMMA 4.3}

Let $\ell$ be an odd prime, and let $p$ be an odd prime such that $p \nmid \ell h_{\ell}^{+}$, where $h_{\ell}^{+}$is the class number of the maximal real subfield of $k$. Then $H_{\mu} / S_{\mu+1}$ is generated by real units of $k$ for all $\mu \in \mathbb{Z}_{>0}$.

\section{Proof}

The $\left(2 \ell h_{\ell}^{+}\right)$th power mapping of $S_{\mu} / S_{\mu+1}$ induces an automorphism of itself because $\operatorname{gcd}\left(p, 2 \ell h_{\ell}^{+}\right)=1$. Thus, the image of $E \cap S_{\mu}$ in $S_{\mu} / S_{\mu+1}$ is the same as that of $E^{2 \ell h_{\ell}^{+}} \cap S_{\mu}$. By Propositions 4.1 and 4.2, $E^{2 \ell h_{\ell}^{+}} \subset\left\langle\xi_{a}^{2 \ell} \mid 1<a<\ell / 2\right\rangle$ where $\xi_{a}=\zeta_{2 \ell}^{1-a}\left(1-\zeta^{a}\right) /(1-\zeta) \in \mathbb{R}$. Therefore, $H_{\mu} / S_{\mu+1}=S_{\mu+1}\left(E^{2 \ell h_{\ell}^{+}} \cap S_{\mu}\right) / S_{\mu+1}$ is generated by real units of $k$.

Let $M_{\ell}(p)=\left[m_{i j}\right] \in M_{(n+1) \times 2 n}(\mathbb{Z} / p \mathbb{Z})$, where $m_{i j}$ is the coefficient of $\zeta^{j}$ in $\varphi^{+}\left(\zeta^{i}\right)$ in $\mathbb{Z} / p \mathbb{Z}$. Then we get

$$
m_{i j}= \begin{cases}1 & \text { if } \bar{i} \cdot \bar{j}^{-1} \in\{\overline{1}, \overline{2}, \ldots, \bar{n}\} \text { in } \mathbb{Z} / \ell \mathbb{Z}, \\ 0 & \text { otherwise }\end{cases}
$$

We can then easily see that the rank of $M_{\ell}(p)$ is equal to the dimension of the vector subspace $\left\langle\eta_{\mu, 1} S_{\mu+1}, \eta_{\mu, 2} S_{\mu+1}, \ldots, \eta_{\mu, n+1} S_{\mu+1}\right\rangle$ in $S_{\mu} / S_{\mu+1}$.

\section{LEMMA 4.4}

Let $\ell$ and $p$ be odd primes, and let $\mu \in \mathbb{Z}_{>0}$. For $1 \leq i, j \leq 2 n$, let

$$
n_{i j}=\left\{\begin{array}{l}
1 \quad \text { if } \bar{i} \cdot \bar{j} \in\{\overline{1}, \overline{2}, \ldots, \bar{n}\} \text { in } \mathbb{Z} / \ell \mathbb{Z}, \\
0 \quad \text { otherwise, }
\end{array}\right.
$$

and let $N_{\ell}=\left[n_{i j}\right]_{1 \leq i, j \leq n} \in M_{n}(\mathbb{Z})$. Then the images $\eta_{\mu, 1}, \eta_{\mu, 2}, \ldots, \eta_{\mu, n+1}$ are linearly independent in $S_{\mu} / S_{\mu+1}$ if and only if $p \nmid \operatorname{det}\left(N_{\ell}\right)$.

\section{Proof}

Let $N_{\ell}^{\prime}=\left[n_{i j}\right]_{1 \leq i \leq n+1,1 \leq j \leq 2 n} \in M_{(n+1) \times 2 n}(\mathbb{Z} / p \mathbb{Z})$. It is clear that $\operatorname{rank}\left(M_{\ell}(p)\right)=$ $\operatorname{rank}\left(N_{\ell}^{\prime}\right)$. Hence, the images $\eta_{\mu, 1}, \eta_{\mu, 2}, \ldots, \eta_{\mu, n+1}$ are linearly independent in $S_{\mu} / S_{\mu+1}$ if and only if $N_{\ell}^{\prime}$ has rank $n+1$. Now, we claim that $\operatorname{rank}\left(N_{\ell}^{\prime}\right)=n+1$ if and only if $N_{\ell}^{\prime}$ induces the following row echelon form:

$$
\left[\begin{array}{cccccccc}
1 & & & & & & & -1 \\
& 1 & & & & & -1 & \\
& & \ddots & & & . \cdot & & \\
& & & 1 & -1 & & & \\
& & & & 1 & 1 & \ldots & 1
\end{array}\right] .
$$

The "if" part is obvious. Note that if $n_{i j}=1$ (resp., 0), then $n_{i \ell-j}=0$ (resp., 1) because there are no two automorphisms among $\varphi_{1}, \varphi_{2}, \ldots, \varphi_{n}$ which are complex 
conjugates of each other. Let $v_{i}$ be the $i$ th row vector of $N_{\ell}^{\prime}$ for $1 \leq i \leq n+1$, and let

$$
v_{i}^{\prime}=\left[v_{i j}^{\prime}\right]_{1 \leq j \leq 2 n}= \begin{cases}2 v_{i}-v_{n}-v_{n+1} & \text { for } 1 \leq i \leq n, \\ v_{n}+v_{n+1} & \text { for } i=n+1 .\end{cases}
$$

Observe that $v_{n+1}^{\prime}=\left[\begin{array}{llll}1 & 1 & \cdots & 1\end{array}\right]$ and $2 \in(\mathbb{Z} / p \mathbb{Z})^{\times}$. For $1 \leq i \leq n$, if $v_{i j}^{\prime}=1$ (resp., -1 ), then $v_{i \ell-j}=-1$ (resp., 1). Thus, we can write $v_{i}^{\prime}$ as a linear combination of the row vectors of the above row echelon form (4.2), and hence, the claim is proved. By the above claim, $\operatorname{rank}\left(N_{\ell}^{\prime}\right)=n+1$ if and only if $\operatorname{det}\left(\left[n_{i j}\right]_{1 \leq i, j \leq n+1}\right) \not \equiv 0$ $(\bmod p)$. Since

$$
\left[-n_{1 j}+n_{n j}+n_{n+1 j}\right]_{1 \leq j \leq n+1}=\left[\begin{array}{lllll}
0 & 0 & \cdots & 0 & 1
\end{array}\right]
$$

we derive

$$
\operatorname{det}\left(\left[n_{i j}\right]_{1 \leq i, j \leq n+1}\right)=\operatorname{det}\left(N_{\ell}\right) .
$$

This completes the proof.

\section{THEOREM 4.5}

Let $\ell$ and $p$ be odd primes, and put $n=(\ell-1) / 2$. Further, let $M_{\ell}(p)$ and $N_{\ell}$ be as above. If $p \nmid \ell h_{\ell}^{+} n$, then for every $\mu \in \mathbb{Z}_{>0}$ we deduce

$$
\operatorname{Gal}\left(K_{\mu} / k_{\mu}\right) \cong(\mathbb{Z} / p \mathbb{Z})^{\operatorname{rank}\left(M_{\ell}(p)\right)} .
$$

If $p \nmid \operatorname{det}\left(N_{\ell}\right)$, then we obtain

$$
\operatorname{Gal}\left(K_{\mu} / k_{\mu}\right) \cong(\mathbb{Z} / p \mathbb{Z})^{n+1}
$$

\section{Proof}

By (4.1) it suffices to show that the dimension of $\left\langle\eta_{\mu, 1} S_{\mu+1}, \eta_{\mu, 2} S_{\mu+1}, \ldots\right.$, $\left.\eta_{\mu, n+1} S_{\mu+1}\right\rangle$ in $S_{\mu} / S_{\mu+1}$ is equal to the dimension of $\left\langle\eta_{\mu, 1} H_{\mu}, \eta_{\mu, 2} H_{\mu}, \ldots\right.$, $\left.\eta_{\mu, n+1} H_{\mu}\right\rangle$ in $S_{\mu} / H_{\mu}$. If $n=1$, then $H_{\mu}=S_{\mu+1}$ by Lemma 4.3 ; hence, we are done in this case. Thus, we may assume $n \geq 2$. It is well known that $\mathbb{Z}\left[\zeta+\zeta^{-1}\right]$ is the ring of integers of the maximal real subfield $\mathbb{Q}\left(\zeta+\zeta^{-1}\right)$ of $k$ and $\left[\mathbb{Q}\left(\zeta+\zeta^{-1}\right)\right.$ : $\mathbb{Q}]=n\left(\right.$ see $\left[16\right.$, Theorem 4]). Therefore, if $u S_{\mu+1} \in H_{\mu} / S_{\mu+1}$, then by Lemma 4.3 we can write

$$
u=1+2 p^{\mu}\left(a_{0}+a_{1}\left(\zeta+\zeta^{-1}\right)+a_{2}\left(\zeta+\zeta^{-1}\right)^{2}+\cdots+a_{n-1}\left(\zeta+\zeta^{-1}\right)^{n-1}\right) \in S_{\mu} \cap E
$$

for some $a_{i} \in \mathbb{Z}$ with $0 \leq i \leq n-1$. If $p \mid a_{i}$ for $1 \leq i \leq n-1$, then

$$
\begin{aligned}
N_{\mathbb{Q}\left(\zeta+\zeta^{-1}\right) / \mathbb{Q}}(u) & \equiv 1+2 p^{\mu} n a_{0} \quad\left(\bmod 2 p^{\mu+1}\right) \\
& \equiv 1 \quad\left(\bmod 2 p^{\mu+1}\right) .
\end{aligned}
$$

Since $p \nmid n$, we have $p \mid a_{0}$ and so $u \in S_{\mu+1}$.

Now, we set

$$
\begin{aligned}
b & =a_{0}+a_{1}\left(\zeta+\zeta^{-1}\right)+a_{2}\left(\zeta+\zeta^{-1}\right)^{2}+\cdots+a_{n-1}\left(\zeta+\zeta^{-1}\right)^{n-1} \\
& =b_{0}+b_{1} \zeta+b_{2} \zeta^{2}+\cdots+b_{n} \zeta^{n}+b_{n} \zeta^{-n}+b_{n-1} \zeta^{-(n-1)}+\cdots+b_{1} \zeta^{-1},
\end{aligned}
$$


where $b_{i} \in \mathbb{Z}$ for $0 \leq i \leq n$. Observe that $b_{n}=0, b_{n-1}=a_{n-1}$, and $b_{n-2}=a_{n-2}$. Consider the following matrix:

$$
\begin{aligned}
& \mathbf{M}=\left[\begin{array}{cccccccc}
1 & & & & & & & -1 \\
& 1 & & & & & -1 & \\
& & \ddots & & & & \\
& & & 1 & -1 & & & \\
b_{1}-b_{0} & b_{2}-b_{0} & \cdots & b_{n}-b_{0} & b_{n}-b_{0} & \cdots & b_{2}-b_{0} & b_{1}-b_{0}
\end{array}\right] \\
& \in M_{(n+2) \times 2 n}(\mathbb{Z} / p \mathbb{Z}) .
\end{aligned}
$$

The last row of $\mathbf{M}$ is induced from the coefficients of $\zeta^{j}$ for $1 \leq j \leq 2 n$ in $b$. So, $\mathbf{M}$ is row equivalent to

$$
\begin{aligned}
& \mathbf{M} \sim\left[\begin{array}{cccccccc}
1 & & & & & & & -1 \\
& 1 & & & & & & \\
& & \ddots & & & & & \\
& & & 1 & -1 & & & \\
& & & & 1 & 1 & \cdots & 1 \\
0 & 0 & \cdots & 0 & 2\left(b_{n}-b_{0}\right) & 2\left(b_{n-1}-b_{0}\right) & \cdots & 2\left(b_{1}-b_{0}\right)
\end{array}\right] \\
& \sim\left[\begin{array}{cccccccc}
1 & & & & & & & -1 \\
& 1 & & & & & -1 & \\
& & \ddots & & & . & & \\
& & & 1 & -1 & & & \\
& & & & 1 & 1 & \cdots & 1 \\
0 & 0 & \cdots & 0 & 0 & 2 b_{n-1} & \cdots & 2 b_{1}
\end{array}\right] \quad \text { because } b_{n}=0 .
\end{aligned}
$$

Here we claim that if $u \notin S_{\mu+1}$, then the rank of $\mathbf{M}$ is $n+2$. Indeed, if $p \nmid a_{n-1}$ or $p \nmid a_{n-2}$, then we are done. Otherwise, we get

$$
\begin{aligned}
& b_{n-3} \equiv a_{n-3} \quad(\bmod p), \\
& b_{n-4} \equiv a_{n-4} \quad(\bmod p) .
\end{aligned}
$$

Hence, by induction we ensure that the rank of $\mathbf{M}$ is $n+1$ if and only if $p \mid a_{i}$ for all $1 \leq i \leq n-1$. Since $u \notin S_{\mu+1}$, we obtain $p \nmid a_{i}$ for some $1 \leq i \leq n-1$, and the claim is proved. In the proof of Lemma 4.4 we already showed that every row vector of $M_{\ell}(p)$ can be written as a linear combination of the row vectors of the matrix (4.2). Therefore, if $u \notin S_{\mu+1}$, then by the above claim for each $1 \leq i \leq n+1$ the images of $\eta_{\mu, i}$ and $u$ in $S_{\mu} / S_{\mu+1}$ are linearly independent, as desired. Furthermore, if $p \nmid \operatorname{det}\left(N_{\ell}\right)$, then by Lemma 4.4 we obtain $\operatorname{rank}\left(M_{\ell}(p)\right)=$ $n+1$.

\section{COROLLARY 4.6}

Suppose that $p \nmid \ell h_{\ell}^{+} n$. Then $K_{\mu}$ becomes the ray class field $k_{\mu+1}$ for all $\mu \in \mathbb{Z}_{>0}$ if and only if $\operatorname{dim}_{\mathbb{Z} / p \mathbb{Z}}\left(H_{1} / S_{2}\right)=n-1$ and $p \nmid \operatorname{det}\left(N_{\ell}\right)$. 
Proof

Suppose that $\operatorname{dim}_{\mathbb{Z} / p \mathbb{Z}}\left(H_{1} / S_{2}\right)=n-1$ and $p \nmid \operatorname{det}\left(N_{\ell}\right)$. We claim that $\operatorname{dim}_{\mathbb{Z} / p \mathbb{Z}}\left(H_{\mu} / S_{\mu+1}\right)=n-1$ for all $\mu \in \mathbb{Z}_{>0}$. Indeed, let $\varepsilon_{1}, \varepsilon_{2}, \ldots, \varepsilon_{n-1}$ be elements of $S_{1} \cap E$ whose images form a basis for $H_{1} / S_{2}$. Since $\varepsilon_{i}^{p} \in H_{2}-S_{3}$ for all $i$, the images $\varepsilon_{1}^{p}, \varepsilon_{2}^{p}, \ldots, \varepsilon_{n-1}^{p}$ turn out to be a basis for $H_{2} / S_{3}$. By induction, the claim is proved. By Theorem 4.5 and the assumptions, we deduce

$$
\begin{aligned}
\left|\operatorname{Gal}\left(K_{\mu} / k_{\mu}\right)\right| & =\left|\widetilde{\varphi_{\mu}^{*}}\left(S_{\mu} / H_{\mu}\right)\right|=p^{n+1}, \\
\left|\operatorname{Gal}\left(k_{\mu+1} / k_{\mu}\right)\right| & =\left|S_{\mu} / H_{\mu}\right|=\frac{\left|S_{\mu} / S_{\mu+1}\right|}{\left|H_{\mu} / S_{\mu+1}\right|}=p^{n+1} .
\end{aligned}
$$

Therefore, $K_{\mu}=k_{\mu+1}$ because $K_{\mu} \subset k_{\mu+1}$.

Conversely, suppose that $K_{\mu}=k_{\mu+1}$ for all $\mu \in \mathbb{Z}_{>0}$. It follows from the proof of Lemma 4.3 that $\left|H_{\mu} / S_{\mu+1}\right| \leq p^{n-1}$, and so $\left|\operatorname{Gal}\left(k_{\mu+1} / k_{\mu}\right)\right| \geq p^{n+1}$. On the other hand, $\left|\operatorname{Gal}\left(K_{\mu} / k_{\mu}\right)\right| \leq p^{n+1}$ by the formula (4.1). Since $K_{\mu}=k_{\mu+1}$, we have $\operatorname{dim}_{\mathbb{Z} / p \mathbb{Z}}\left(H_{1} / S_{2}\right)=n-1$ and $\left|\operatorname{Gal}\left(K_{\mu} / k_{\mu}\right)\right|=p^{n+1}$; hence $p \nmid \operatorname{det}\left(N_{\ell}\right)$ by Lemma 4.4 and Theorem 4.5.

\section{REMARK 4.7}

(i) We are able to show that $\operatorname{det}\left(N_{\ell}\right) \neq 0$ for $\ell \leq 10000$ with the aid of Maple software, from which we conjecture that $\operatorname{det}\left(N_{\ell}\right) \neq 0$ holds for all odd primes $\ell$.

(ii) Let $\xi_{a}=\zeta_{2 \ell}^{1-a}\left(1-\zeta^{a}\right) /(1-\zeta) \in \mathbb{R}$ for $1<a<\ell / 2$. When $\ell=5$, we obtain

$$
\begin{aligned}
\xi_{2}^{48} & \equiv 1 \quad(\bmod 14) \\
& \neq \equiv 1 \quad(\bmod 98) .
\end{aligned}
$$

Thus, $\operatorname{dim}_{\mathbb{Z} / 7 \mathbb{Z}}\left(H_{1} / S_{2}\right)=1$, and Corollary 4.6 is true for $p=7$. In a similar way, we can show that $\operatorname{dim}_{\mathbb{Z} / p \mathbb{Z}}\left(H_{1} / S_{2}\right)=1$ holds for all odd primes $p \leq 101$ except $p=3$. When $\ell=7$, we see that $\operatorname{dim}_{\mathbb{Z} / p \mathbb{Z}}\left(H_{1} / S_{2}\right)=2$ for all odd primes $p \leq 101$. So, we also conjecture that, for each odd prime $\ell, \operatorname{dim}_{\mathbb{Z} / p \mathbb{Z}}\left(H_{1} / S_{2}\right)=n-1$ holds for almost all odd primes $p$. If the above two conjectures are true, then we have the isomorphism

$$
\operatorname{Gal}\left(k_{\mu+1} / k_{\mu}\right) \cong(\mathbb{Z} / p \mathbb{Z})^{n+1}
$$

for each odd prime $\ell$ and almost all odd primes $p$.

(iii) Table 1 lists the generators of $H_{1} / S_{2}$ for $\ell=5,7$.

\section{Theta functions}

In this section, we shall provide necessary fundamental transformation formulas of theta functions and describe the action of $G_{\mathbb{A}+}$ on the quotient of two theta constants.

Let $n$ be a positive integer, and let $u \in \mathbb{C}^{n}, z \in \mathbb{H}_{n}$, and $r, s \in \mathbb{R}^{n}$. We define a (classical) theta function by

$$
\Theta(u, z ; r, s)=\sum_{x \in \mathbb{Z}^{n}} e\left(\frac{1}{2} \cdot{ }^{t}(x+r) z(x+r)+{ }^{t}(x+r)(u+s)\right) .
$$


Table 1

\begin{tabular}{|r|c|c|}
\hline \multirow{2}{*}{$p$} & \multicolumn{2}{|c|}{ generators of $H_{1} / S_{2}$} \\
\cline { 2 - 3 } & $\ell=5$ & $\ell=7$ \\
\hline 3 & 1 & $\xi_{2}^{182}, \xi_{3}^{182}$ \\
\hline 5 & $\xi_{2}^{60}$ & $\xi_{2}^{868}, \xi_{3}^{868}$ \\
\hline 7 & $\xi_{2}^{48}$ & $\xi_{2}^{42}, \xi_{3}^{42}$ \\
\hline 11 & $\xi_{2}^{30}$ & $\xi_{2}^{1330}, \xi_{3}^{1330}$ \\
\hline 13 & $\xi_{2}^{84}$ & $\xi_{2}^{84}, \xi_{3}^{84}$ \\
\hline 17 & $\xi_{2}^{72}$ & $\xi_{2}^{17192}, \xi_{3}^{34384}$ \\
\hline 19 & $\xi_{2}^{18}$ & $\xi_{2}^{16002}, \xi_{3}^{16002}$ \\
\hline 23 & $\xi_{2}^{528}$ & $\xi_{2}^{12166}, \xi_{3}^{12166}$ \\
\hline 29 & $\xi_{2}^{848}$ & $\xi_{2}^{28}, \xi_{3}^{28}$ \\
\hline 31 & $\xi_{2}^{30}$ & $\xi_{2}^{69510}, \xi_{3}^{69510}$ \\
\hline 37 & $\xi_{2}^{684}$ & $\xi_{2}^{16884}, \xi_{3}^{16884}$ \\
\hline 41 & $\xi_{2}^{120}$ & $\xi_{2}^{280}, \xi_{3}^{280}$ \\
\hline 43 & $\xi_{2}^{1848}$ & $\xi_{2}^{42}, \xi_{3}^{42}$ \\
\hline
\end{tabular}

\begin{tabular}{|r|c|c|}
\hline \multirow{2}{*}{$p$} & \multicolumn{2}{|c|}{ generators of $H_{1} / S_{2}$} \\
\cline { 2 - 3 } & $\ell=5$ & $\ell=7$ \\
\hline 47 & $\xi_{2}^{2208}$ & $\xi_{2}^{726754}, \xi_{3}^{726754}$ \\
\hline 53 & $\xi_{2}^{1404}$ & $\xi_{2}^{148876}, \xi_{3}^{148876}$ \\
\hline 59 & $\xi_{2}^{174}$ & $\xi_{2}^{1437646}, \xi_{3}^{1437646}$ \\
\hline 61 & $\xi_{2}^{60}$ & $\xi_{2}^{40740}, \xi_{3}^{40740}$ \\
\hline 67 & $\xi_{2}^{4488}$ & $\xi_{2}^{100254}, \xi_{3}^{100254}$ \\
\hline 71 & $\xi_{2}^{210}$ & $\xi_{2}^{70}, \xi_{3}^{70}$ \\
\hline 73 & $\xi_{2}^{1332}$ & $\xi_{2}^{226926}, \xi_{3}^{453852}$ \\
\hline 79 & $\xi_{2}^{78}$ & $\xi_{2}^{164346}, \xi_{3}^{164346}$ \\
\hline 83 & $\xi_{2}^{6888}$ & $\xi_{2}^{574}, \xi_{3}^{574}$ \\
\hline 89 & $\xi_{2}^{132}$ & $\xi_{2}^{1233694}, \xi_{3}^{4934776}$ \\
\hline 97 & $\xi_{2}^{2352}$ & $\xi_{2}^{672}, \xi_{3}^{672}$ \\
\hline 101 & $\xi_{2}^{300}$ & $\xi_{2}^{7212100}, \xi_{3}^{7212100}$ \\
\hline
\end{tabular}

PROPOSITION 5.1

Let $r, s \in \mathbb{R}^{n}$ and $a, b \in \mathbb{Z}^{n}$. We have

(i) $\Theta(-u, z ;-r,-s)=\Theta(u, z ; r, s)$,

(ii) $\Theta(u, z ; r+a, s+b)=e\left({ }^{t} r b\right) \Theta(u, z ; r, s)$.

Proof

See $[26$, p. 676, (13)].

For a square matrix $S$, by $\{S\}$ we mean the column vector whose components are the diagonal entries of $S$.

\section{PROPOSITION 5.2}

For every $\gamma=\left[\begin{array}{ll}A & B \\ C & D\end{array}\right] \in \Gamma(1)$ such that $\left\{{ }^{t} A C\right\},\left\{{ }^{t} B D\right\} \in 2 \mathbb{Z}^{n}$, we get the transformation formula

$$
\begin{aligned}
& \Theta\left({ }^{t}(C z+D)^{-1} u, \gamma(z) ; r, s\right) \\
& =\lambda_{\gamma} e\left(\frac{{ }^{t} r s-{ }^{t} r^{\prime} s^{\prime}}{2}\right) \operatorname{det}(C z+D)^{1 / 2} e\left(\frac{1}{2} \cdot{ }^{t} u(C z+D)^{-1} c u\right) \\
& \quad \times \Theta\left(u, z ; r^{\prime}, s^{\prime}\right),
\end{aligned}
$$

where $\lambda_{\gamma}$ is a constant of absolute value 1 depending only on $\gamma$ and the choice of the branch of $\operatorname{det}(C z+D)^{1 / 2}$, and

$$
\left[\begin{array}{l}
r^{\prime} \\
s^{\prime}
\end{array}\right]={ }^{t} \gamma\left[\begin{array}{l}
r \\
s
\end{array}\right] .
$$

In particular, $\lambda_{\gamma}^{4}=1$ for $\gamma \in \Gamma(2)$. 
Proof

See [26, Propositions 1.3 and 1.4].

Here, the functions $\Theta(0, z ; r, s)$ are called theta constants, and these are holomorphic on $\mathbb{H}_{n}$ as functions in $z$ (see [26, Proposition 1.6]).

\section{PROPOSITION 5.3}

Suppose that $r, s$ belong to $\mathbb{Q}^{n}$. Then the theta constant $\Theta(0, z ; r, s)$ represents the zero function if and only if $r, s \in(1 / 2) \mathbb{Z}^{n}$ and $e\left(2 \cdot{ }^{t} r s\right)=-1$.

Proof

See [11, Theorem 2].

Let

$$
\Phi_{(r, s)}(z)=\frac{\Theta(0, z ; r, s)}{\Theta(0, z ; 0,0)} .
$$

Note that the poles of $\Phi_{(r, s)}(z)$ are exactly the zeros of $\Theta(0, z ; 0,0)=$ $\sum_{x \in \mathbb{Z}^{n}} e\left(\frac{1}{2}^{t} x z x\right)$. When $n=1, \Theta(0, z ; 0,0)$ has no zero on $\mathbb{H}_{1}$ by Jacobi's triple product identity (see [1, Theorem 14.6]).

\section{LEMMA 5.4}

For $r, s \in \mathbb{R}^{n}$ and $a, b \in \mathbb{Z}^{n}$, we obtain that

(i) $\Phi_{(-r,-s)}(z)=\Phi_{(r, s)}(z)$,

(ii) $\Phi_{(r+a, s+b)}(z)=e\left({ }^{t} r b\right) \Phi_{(r, s)}(z)$,

(iii) if $\gamma=\left[\begin{array}{cc}A & B \\ C & D\end{array}\right] \in \Gamma(1)$ such that $\left\{{ }^{t} A C\right\},\left\{{ }^{t} B D\right\} \in 2 \mathbb{Z}^{n}$, then we obtain

$$
\Phi_{(r, s)}(\gamma(z))=e\left(\frac{{ }^{t} r s-{ }^{t} r^{\prime} s^{\prime}}{2}\right) \Phi_{\left(r^{\prime}, s^{\prime}\right)}(z),
$$

where

$$
\left[\begin{array}{l}
r^{\prime} \\
s^{\prime}
\end{array}\right]={ }^{t} \gamma\left[\begin{array}{l}
r \\
s
\end{array}\right]
$$

Proof

It is immediate from Propositions 5.1 and 5.2.

PROPOSITION 5.5

Let $m$ be a positive integer, and let $r, s \in(1 / m) \mathbb{Z}^{n}$. Then $\Phi_{(r, s)}(z)$ belongs to $\mathcal{F}_{2 m^{2}}$. Moreover, if $x$ is an element of $\Delta$ such that

$$
x_{q} \equiv\left[\begin{array}{cc}
1_{n} & 0 \\
0 & t 1_{n}
\end{array}\right] \quad\left(\bmod 2 m^{2} M_{2 n}\left(\mathbb{Z}_{q}\right)\right)
$$

for all rational primes $q$ and a positive integer $t$, then

$$
\Phi_{(r, s)}(z)^{x}=\Phi_{(r, t s)}(z) .
$$


Proof

See [26, Proposition 1.7].

COROLLARY 5.6

For $m \in \mathbb{Z}_{>0}$ and $r, s \in(1 / m) \mathbb{Z}^{n}$, let

$$
y=\beta\left[\begin{array}{cc}
1_{n} & 0 \\
0 & x \cdot 1_{n}
\end{array}\right] \alpha \in G_{\mathbb{A}+}
$$

with $\beta \in R_{2 m^{2}}, x \in \prod_{q} \mathbb{Z}_{q}^{\times}$, and $\alpha \in G_{\mathbb{Q}+}$. Then

$$
\left(\Phi_{(r, s)}\right)^{y}(z)=\Phi_{(r, t s)}(\alpha(z)),
$$

where $t$ is a positive integer such that $t \equiv x_{q}\left(\bmod 2 m^{2} \mathbb{Z}_{q}\right)$ for all rational primes $q$.

Proof

This can be proved by Propositions 2.2 and 5.5 .

\section{Construction of class fields}

We use the same notation as in Section 4 . Let $k=\mathbb{Q}(\zeta)$ with $\zeta=\zeta_{\ell}$, and let $n=(\ell-1) / 2$ so that $2 n=[k: \mathbb{Q}]$. Let $v: k \rightarrow \mathbb{C}^{n}$ be the map given by

$$
v(x)=\left[\begin{array}{c}
x^{\varphi_{1}} \\
\vdots \\
x^{\varphi_{n}}
\end{array}\right]
$$

let $L=v\left(\mathcal{O}_{k}\right)$ be a lattice in $\mathbb{C}^{n}$, and let $\rho=\left(\zeta-\zeta^{-1}\right) / \ell \in k$. Then $\rho$ satisfies conditions (i)-(iii) in Section 3. We have an $\mathbb{R}$-bilinear form $E: \mathbb{C}^{n} \times \mathbb{C}^{n} \rightarrow \mathbb{R}$ defined by

$$
E(z, w)=\sum_{i=1}^{n} \rho^{\varphi_{i}}\left(z_{i} \overline{w_{i}}-\overline{z_{i}} w_{i}\right) \quad \text { for } z=\left[\begin{array}{c}
z_{1} \\
\vdots \\
z_{n}
\end{array}\right], w=\left[\begin{array}{c}
w_{1} \\
\vdots \\
w_{n}
\end{array}\right],
$$

which induces a nondegenerate Riemann form on $\mathbb{C}^{n} / L$. Let

$$
e_{i}= \begin{cases}\zeta^{2 i} & \text { for } 1 \leq i \leq n, \\ \sum_{j=1}^{i-n} \zeta^{2 j-1} & \text { for } n+1 \leq i \leq 2 n\end{cases}
$$

Since $\left\{e_{1}, e_{2}, \ldots, e_{2 n}\right\}$ is a free $\mathbb{Z}$-basis of $\mathcal{O}_{k},\left\{v\left(e_{1}\right), v\left(e_{2}\right), \ldots, v\left(e_{2 n}\right)\right\}$ is a free $\mathbb{Z}$-basis of the lattice $L$, and we get

$$
\left[E\left(v\left(e_{i}\right), v\left(e_{j}\right)\right)\right]_{1 \leq i, j \leq 2 n}=J .
$$

Now, let

$$
\Omega=\left[\begin{array}{llll}
v\left(e_{1}\right) & v\left(e_{2}\right) \cdots & \cdots\left(e_{2 n}\right)
\end{array}\right] \in M_{n \times 2 n}(\mathbb{C}) .
$$


Then $\Omega$ satisfies

$$
\begin{aligned}
L & =\left\{\Omega\left[\begin{array}{l}
a \\
b
\end{array}\right] \mid a, b \in \mathbb{Z}^{n}\right\}, \\
E(\Omega x, \Omega y) & ={ }^{t} x J y \quad \text { for } x, y \in \mathbb{R}^{2 n},
\end{aligned}
$$

because $E$ is $\mathbb{R}$-bilinear. Thus, $\delta=1$ and $\epsilon=1_{n}$ in Section 3. Write $\Omega=\left[\Omega_{1} \Omega_{2}\right]$ with $\Omega_{1}, \Omega_{2} \in M_{n}(\mathbb{C})$, and put $z_{\ell}=\Omega_{2}^{-1} \Omega_{1} \in \mathbb{H}_{n}$. We define a ring monomorphism $h$ of $k_{\mathbb{A}}$ into $M_{2 n}\left(\mathbb{Q}_{\mathbb{A}}\right)$ as in Section 3. Then $z_{\ell}$ is the CM-point of $\mathbb{H}_{n}$ induced from $h$ corresponding to the polarized abelian variety $\left(\mathbb{C}^{n} / L, E\right)$.

Let $p$ be an odd prime, and let $r, s \in \mathbb{Q}^{n}$. We denote by $\mathbf{h}$ the set of all non-Archimedean primes of $k$ and by a the set of all archimedean primes of $k$. For given $\omega \in \mathcal{O}_{k}$ prime to $2 p$, we set

$$
\widetilde{\omega}=\prod_{\substack{v \in \mathbf{h} \\ v \mid 2 p}}\left(\omega^{-1}\right)_{v} \times \prod_{\substack{v \in \mathbf{h} \\ v \nmid 2 p}} 1_{v} \times \prod_{v \in \mathbf{a}} 1_{v} \in k_{\mathbb{A}}^{\times} .
$$

Here $x_{v}$ is the $v$-component of $x \in k_{\mathbb{A}}^{\times}$. If $\Phi_{(r, s)}$ is finite at $z_{\ell}$, then by Proposition 3.2 and [15, Chapter 8, Section 4], we have

$$
\Phi_{(r, s)}\left(z_{\ell}\right)^{\left(\frac{k^{\prime} / k}{(\omega)}\right)}=\Phi_{(r, s)}\left(z_{\ell}\right)^{[\widetilde{\omega}, k]}=\left(\Phi_{(r, s)}\right)^{h\left(\varphi^{*}\left(\widetilde{\omega}^{-1}\right)\right)}\left(z_{\ell}\right) \quad \text { for } \omega \in \mathcal{O}_{k},
$$

where $k^{\prime}$ is a finite abelian extension of $k$ containing $\Phi_{(r, s)}\left(z_{\ell}\right)$.

\section{LEMMA 6.1}

Let $p$ be an odd prime, let $\mu \in \mathbb{Z}_{>0}$, let $r, s \in\left(1 / p^{\mu}\right) \mathbb{Z}^{n}$, and let $z_{\ell}$ be as above. Assume that $z_{\ell}$ is not a zero of $\Theta(0, z ; 0,0)$. If $p \nmid \ell h_{\ell}^{+} n$, then $\Phi_{(r, s)}\left(z_{\ell}\right)^{p^{\alpha}} \in$ $K_{2 \mu-1-\alpha}$ for $\alpha=0,1, \ldots, \mu$.

\section{Proof}

By Proposition 5.5 and [26, p. 682], $\Phi_{(r, s)}(z)$ belongs to $\mathcal{F}_{2 p^{2 \mu}}$ as a function, so

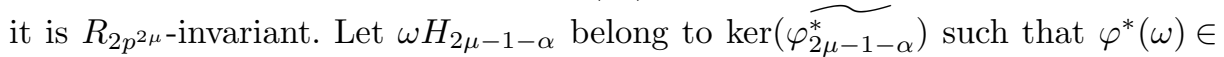
$H_{2 \mu-1-\alpha}$. Since $p \nmid \ell h_{\ell}^{+} n$, it follows from the proof of Theorem 4.5 that $\left(\varphi^{*}\left(S_{2 \mu-1-\alpha}\right) \cap H_{2 \mu-1-\alpha}\right) / S_{2 \mu-\alpha}=\{0\}$. Hence, $\varphi^{*}(\omega) \in S_{2 \mu-\alpha}$. Write $\varphi^{*}(\omega)=$ $1+2 p^{2 \mu-\alpha} \omega_{0}$ with $\omega_{0} \in \mathcal{O}_{k}$. Then it suffices to show that $\left(\Phi_{(r, s)}\left(z_{\ell}\right)^{p^{\alpha}}\right)^{\left(\frac{k^{\prime} / k}{(\omega)}\right)}=$ $\Phi_{(r, s)}\left(z_{\ell}\right)^{p^{\alpha}}$, where $k^{\prime}$ is a finite abelian extension of $k$ containing $\Phi_{(r, s)}\left(z_{\ell}\right)$. By the strong approximation theorem for $\operatorname{Sp}(n)$ there exists a matrix $\beta \in \Gamma(1)$ such that

$$
h\left(\varphi^{*}(\omega)\right) \equiv\left[\begin{array}{cc}
1_{n} & 0 \\
0 & v \cdot 1_{n}
\end{array}\right] \beta \quad\left(\bmod 2 p^{2 \mu}\right),
$$

where $v:=\nu\left(h\left(\varphi^{*}(\omega)\right)\right)=N_{k / \mathbb{Q}}(\omega)=\varphi^{*}(\omega) \cdot \overline{\varphi^{*}(\omega)}=1+2 p^{2 \mu-\alpha} v_{0}$ for some $v_{0} \in$ $\mathbb{Z}$. In fact, $\beta$ belongs to $\Gamma(2)$ because $h\left(\varphi^{*}(\omega)\right) \equiv 1_{2 n}(\bmod 2)$ and $v \equiv 1(\bmod 2)$. Thus, for all rational primes $q$ we obtain

$$
h\left(\varphi^{*}\left(\widetilde{\omega}^{-1}\right)\right)_{q} \equiv\left[\begin{array}{cc}
1_{n} & 0 \\
0 & v \cdot 1_{n}
\end{array}\right] \beta \quad\left(\bmod 2 p^{2 \mu} M_{2 n}\left(\mathbb{Z}_{q}\right)\right) .
$$


By Lemma 5.4 and Corollary 5.6 we get that

$$
\begin{aligned}
\left(\Phi_{(r, s)}\right)^{h\left(\varphi^{*}\left(\widetilde{\omega}^{-1}\right)\right)}\left(z_{\ell}\right) & =\Phi_{(r, v s)}\left(\beta z_{\ell}\right) \\
& =e\left(\frac{{ }^{t} r v s-{ }^{t} r^{\prime} s^{\prime}}{2}\right) \Phi_{\left(r^{\prime}, s^{\prime}\right)}\left(z_{\ell}\right),
\end{aligned}
$$

where

$$
\begin{aligned}
{\left[\begin{array}{l}
r^{\prime} \\
s^{\prime}
\end{array}\right] } & ={ }^{t} \beta\left[\begin{array}{c}
r \\
v s
\end{array}\right] \equiv{ }^{t} h\left(\varphi^{*}(\omega)\right)\left[\begin{array}{l}
r \\
s
\end{array}\right] \quad\left(\bmod 2 p^{2 \mu}\right) \\
& \equiv\left[\begin{array}{l}
r \\
s
\end{array}\right]+2 p^{2 \mu-\alpha} \cdot{ }^{t} h\left(\omega_{0}\right)\left[\begin{array}{l}
r \\
s
\end{array}\right] \quad\left(\bmod 2 p^{2 \mu}\right) .
\end{aligned}
$$

Let $a, b \in 2 p^{\mu-\alpha} \mathbb{Z}^{n} \subset 2 \mathbb{Z}^{n}$ such that $\left[\begin{array}{l}a \\ b\end{array}\right]=2 p^{2 \mu-\alpha} \cdot{ }^{t} h\left(\omega_{0}\right)\left[\begin{array}{l}r \\ s\end{array}\right]$. Then we derive by Lemma 5.4 and Proposition 3.2 that

$$
\begin{aligned}
\left(\Phi_{(r, s)}\left(z_{\ell}\right)^{p^{\alpha}}\right)^{\left(\frac{k^{\prime} / k}{(\omega)}\right)} & =\left(\Phi_{(r, s)} h\left(\varphi^{*}\left(\widetilde{\omega}^{-1}\right)\right)\left(z_{\ell}\right)\right)^{p^{\alpha}} \\
& =\left(e\left(\frac{{ }^{t} r v s-{ }^{t}(r+a)(s+b)}{2}\right) e\left({ }^{t} r b\right) \Phi_{(r, s)}\left(z_{\ell}\right)\right)^{p^{\alpha}} \\
& =e\left(p^{2 \mu} v_{0} \cdot{ }^{t} r s\right) e\left(p^{\alpha} \cdot \frac{{ }^{t} r b-{ }^{t} a s}{2}\right) \Phi_{(r, s)}\left(z_{\ell}\right)^{p^{\alpha}} \\
& =\Phi_{(r, s)}\left(z_{\ell}\right)^{p^{\alpha}} .
\end{aligned}
$$

Let $\mu \in \mathbb{Z}_{>0}$. Assume that $r, s \in\left(1 / p^{\mu}\right) \mathbb{Z}^{n}$ and that $z_{\ell}$ is not a zero of $\Theta(0, z ; 0,0)$. Consider the matrices $h\left(\varphi^{*}\left(\omega_{2 \mu-1-\alpha, j}\right)\right)$ for $1 \leq j \leq n+1$ and $0 \leq \alpha \leq \mu-1$. Then we have

$$
\begin{aligned}
h\left(\varphi^{*}\left(\omega_{2 \mu-1-\alpha, j}\right)\right) & =h\left(1+2 p^{2 \mu-1-\alpha} \varphi^{+}\left(\zeta^{j}\right)+2 p^{2 \mu-\alpha} \omega_{0}\right) \\
& =1_{2 n}+2 p^{2 \mu-1-\alpha} h\left(\varphi^{+}\left(\zeta^{j}\right)\right)+2 p^{2 \mu-\alpha} h\left(\omega_{0}\right)
\end{aligned}
$$

for some $\omega_{0} \in \mathcal{O}_{k}$. Also, we can deduce without difficulty

$$
h(\zeta)=\left[\begin{array}{ccccc|ccccc}
0 & 0 & \cdots & \cdots & 0 & -1 & 1 & & & \\
\vdots & \vdots & & & \vdots & & -1 & 1 & & \\
\vdots & \vdots & & & \vdots & & & \ddots & \ddots & \\
0 & 0 & \cdots & \cdots & 0 & & & & -1 & 1 \\
-1 & -1 & \cdots & \cdots & -1 & & & & & -1 \\
\hline 1 & & & & & 0 & 0 & \cdots & \cdots & 0 \\
1 & 1 & & & & \vdots & \vdots & & & \vdots \\
1 & 1 & 1 & & & \vdots & \vdots & & & \vdots \\
\vdots & \vdots & & \ddots & & \vdots & \vdots & & & \vdots \\
1 & 1 & 1 & \cdots & 1 & 0 & 0 & \cdots & \cdots & 0
\end{array}\right] .
$$


Now, again by the strong approximation theorem for $\operatorname{Sp}(n)$ there exists a matrix $\beta_{2 \mu-1-\alpha, j}$ in $\Gamma(1)$ such that

$$
h\left(\varphi^{*}\left(\omega_{2 \mu-1-\alpha, j}\right)\right) \equiv\left[\begin{array}{cc}
1_{n} & 0 \\
0 & v_{2 \mu-1-\alpha, j} \cdot 1_{n}
\end{array}\right] \beta_{2 \mu-1-\alpha, j} \quad\left(\bmod 2 p^{2 \mu}\right),
$$

where $v_{2 \mu-1-\alpha, j}:=\nu\left(h\left(\varphi^{*}\left(\omega_{2 \mu-1-\alpha, j}\right)\right)\right)=N_{k / \mathbb{Q}}\left(\omega_{2 \mu-1-\alpha, j}\right)=1-2 p^{2 \mu-1-\alpha}+$ $2 p^{2 \mu-\alpha} v_{j}$ for some $v_{j} \in \mathbb{Z}$. As a matter of fact, $\beta_{2 \mu-1-\alpha, j}$ belongs to $\Gamma(2)$ because $h\left(\varphi^{*}\left(\omega_{2 \mu-1-\alpha, j}\right)\right) \equiv 1_{2 n}(\bmod 2)$ and $v_{2 \mu-1-\alpha, j} \equiv 1(\bmod 2)$. For all rational primes $q$ we obtain

$$
h\left(\varphi^{*}\left(\widetilde{\omega}_{2 \mu-1-\alpha, j}^{-1}\right)\right)_{q} \equiv\left[\begin{array}{cc}
1_{n} & 0 \\
0 & v_{2 \mu-1-\alpha, j} \cdot 1_{n}
\end{array}\right] \beta_{2 \mu-1-\alpha, j} \quad\left(\bmod 2 p^{2 \mu} M_{2 n}\left(\mathbb{Z}_{q}\right)\right) .
$$

By Lemma 5.4 and Corollary 5.6 we obtain

$$
\begin{aligned}
& \Phi_{(r, s)} h\left(\varphi^{*}\left(\widetilde{\omega}_{2 \mu-1-\alpha, j}^{-1}\right)\right)\left(z_{\ell}\right)=\Phi_{\left(r, v_{2 \mu-1-\alpha, j} s\right)}\left(\beta_{2 \mu-1-\alpha, j}\left(z_{\ell}\right)\right) \\
& =e\left(\frac{{ }^{t} r v_{2 \mu-1-\alpha, j} s-{ }^{t} r^{\prime} s^{\prime}}{2}\right) \Phi_{\left(r^{\prime}, s^{\prime}\right)}\left(z_{\ell}\right),
\end{aligned}
$$

where

$$
\begin{aligned}
{\left[\begin{array}{l}
r^{\prime} \\
s^{\prime}
\end{array}\right] } & ={ }^{t} \beta_{2 \mu-1-\alpha, j}\left[\begin{array}{c}
r \\
v_{2 \mu-1-\alpha, j} s
\end{array}\right] \\
& \equiv{ }^{t} h\left(\varphi^{*}\left(\omega_{2 \mu-1-\alpha, j}\right)\right)\left[\begin{array}{l}
r \\
s
\end{array}\right] \quad\left(\bmod 2 p^{2 \mu}\right) \\
& \equiv\left[\begin{array}{l}
r \\
s
\end{array}\right]+2 p^{2 \mu-1-\alpha} \cdot{ }^{t} h\left(\varphi^{+}\left(\zeta^{j}\right)\right)\left[\begin{array}{l}
r \\
s
\end{array}\right]+2 p^{2 \mu-\alpha} \cdot{ }^{t} h\left(\omega_{0}\right)\left[\begin{array}{l}
r \\
s
\end{array}\right] \quad\left(\bmod 2 p^{2 \mu}\right) .
\end{aligned}
$$

For each $j$, let $a_{2 \mu-1-\alpha, j}, b_{2 \mu-1-\alpha, j} \in 2 p^{\mu-1-\alpha} \mathbb{Z}^{n} \subset 2 \mathbb{Z}^{n}$ such that

$$
\left[\begin{array}{l}
a_{2 \mu-1-\alpha, j} \\
b_{2 \mu-1-\alpha, j}
\end{array}\right]=2 p^{2 \mu-1-\alpha} \cdot{ }^{t} h\left(\varphi^{+}\left(\zeta^{j}\right)\right)\left[\begin{array}{l}
r \\
s
\end{array}\right] .
$$

Let $c, d \in 2 p^{\mu-\alpha} \mathbb{Z}^{n} \subset 2 \mathbb{Z}^{n}$ for which $\left[\begin{array}{l}c \\ d\end{array}\right]=2 p^{2 \mu-\alpha} \cdot{ }^{t} h\left(\omega_{0}\right)\left[\begin{array}{l}r \\ s\end{array}\right]$. Then by Lemma 5.4 and Proposition 3.2 we derive that

$$
\begin{aligned}
& \left(\Phi_{(r, s)}\left(z_{\ell}\right)^{p^{\alpha}}\right)^{\left(\frac{K_{2 \mu-1-\alpha} / k}{\left(\omega_{2 \mu-1-\alpha, j}\right)}\right)} \\
& =\left(\Phi_{(r, s)} h\left(\varphi^{*}\left(\widetilde{\omega}_{2 \mu-1-\alpha, j}^{-1}\right)\right)\left(z_{\ell}\right)\right)^{p^{\alpha}} \\
& =e\left(p^{\alpha} \frac{{ }^{t} r v_{2 \mu-1-\alpha, j} s-{ }^{t}\left(r+a_{2 \mu-1-\alpha, j}+c\right)\left(s+b_{2 \mu-1-\alpha, j}+d\right)}{2}\right) \\
& \times e\left(p^{\alpha} \cdot{ }^{t} r\left(b_{2 \mu-1-\alpha, j}+d\right)\right) \Phi_{(r, s)}\left(z_{\ell}\right)^{p^{\alpha}} \\
& =e\left(-p^{2 \mu-1} \cdot{ }^{t} r s\right) e\left(p^{\alpha} \frac{{ }^{t} r b_{2 \mu-1-\alpha, j}-{ }^{t} a_{2 \mu-1-\alpha, j} s}{2}\right) \Phi_{(r, s)}\left(z_{\ell}\right)^{p^{\alpha}} \\
& =\underbrace{e\left(-p^{2 \mu-1} \cdot{ }^{t} r s\right) e\left(\frac{{ }^{t} r b_{2 \mu-1, j}-{ }^{t} a_{2 \mu-1, j} s}{2}\right)}_{p \text { th root of unity }} \Phi_{(r, s)}\left(z_{\ell}\right)^{p^{\alpha}} .
\end{aligned}
$$


For given $r_{i}, s_{i} \in \mathbb{Z}^{n}$ with $1 \leq i \leq n+1$, let $\mathbf{r}=\left[r_{i}\right]_{1 \leq i \leq n+1}$ and $\mathbf{s}=\left[s_{i}\right]_{1 \leq i \leq n+1}$. We set $\Phi_{[\mathbf{r}, \mathbf{s} ; \mu, i]}(z)_{p}=\Phi_{\left(r_{i} / p^{\mu}, s_{i} / p^{\mu}\right)}(z)$ for each $\mu \in \mathbb{Z}_{>0}$.

\section{LEMMA 6.2}

Let $\mathbf{r}=\left[r_{i}\right]_{1 \leq i \leq n+1}$ and $\mathbf{s}=\left[s_{i}\right]_{1 \leq i \leq n+1}$ for given $r_{i}, s_{i} \in \mathbb{Z}^{n}$. Then for each $1 \leq$ $i, j \leq n+1$, there exists an integer $c_{i j}$ such that

$$
\Phi_{[\mathbf{r}, \mathbf{s} ; 1, i]}\left(z_{\ell}\right)_{p}^{\left(\frac{K_{1} / k}{\left(\omega_{1, j}\right)}\right)}=\zeta_{p}^{c_{i j}} \Phi_{[\mathbf{r}, \mathbf{s} ; 1, i]}\left(z_{\ell}\right)_{p}
$$

for all odd primes $p$.

Proof

Substituting $r=r_{i} / p$ and $s=s_{i} / p$ into the formula (6.1) we get

$$
\begin{aligned}
\Phi_{[\mathbf{r}, \mathbf{s} ; 1, i]}\left(z_{\ell}\right)_{p}^{\left(\frac{K_{1} / k}{\left(\omega_{1, j}\right)}\right)} & =e\left(-\frac{{ }^{t} r_{i} s_{i}}{p}\right) e\left(\frac{{ }^{t} r_{i} b_{1, j}-{ }^{t} a_{1, j} s_{i}}{2 p}\right) \Phi_{[\mathbf{r}, \mathbf{s} ; 1, i]}\left(z_{\ell}\right)_{p} \\
& =\zeta_{p}^{-{ }^{t} r_{i} s_{i}+\left({ }^{t} r_{i} b_{1, j}-{ }^{t} a_{1, j} s_{i}\right) / 2} \Phi_{[\mathbf{r}, \mathbf{s} ; 1, i]}\left(z_{\ell}\right)_{p},
\end{aligned}
$$

where $a_{1, j}, b_{1, j} \in 2 \mathbb{Z}^{n}$ such that $\left[\begin{array}{l}a_{1, j} \\ b_{1, j}\end{array}\right]=2 \cdot{ }^{t} h\left(\varphi^{+}\left(\zeta^{j}\right)\right)\left[\begin{array}{c}r_{i} \\ s_{i}\end{array}\right]$. Hence,

$$
c_{i j}:=-{ }^{t} r_{i} s_{i}+\frac{1}{2}\left({ }^{t} r_{i} b_{1, j}-{ }^{t} a_{1, j} s_{i}\right)
$$

is an integer which does not depend on $p$.

We put $A_{\ell}(\mathbf{r}, \mathbf{s})=\left[c_{i j}\right]_{1 \leq i, j \leq n+1} \in M_{n+1}(\mathbb{Z})$, where $c_{i j}$ is an integer satisfying

$$
\Phi_{[\mathbf{r}, \mathbf{s} ; 1, i]}\left(z_{\ell}\right)_{p}^{\left(\frac{K_{1} / k}{\left(\omega_{1, j}\right)}\right)}=\zeta_{p}^{c_{i j}} \Phi_{[\mathbf{r}, \mathbf{s} ; 1, i]}\left(z_{\ell}\right)_{p}
$$

for all odd primes $p$. Note that $A_{\ell}(\mathbf{r}, \mathbf{s})$ does not depend on $p$ by Lemma 6.2.

Now, in order to construct a primitive generator of $K_{\mu}$ over $k_{\mu}$, we need the following lemma.

\section{LEMMA 6.3}

Let $L$ be an abelian extension of a number field $F$. Suppose that $L=F(\alpha, \beta)$ for some $\alpha, \beta \in L$. Let $a, b$ be any nonzero elements of $F$, and let $\nu=[L: F(\alpha)]$. Then we have

$$
L=F\left(a \alpha+b\left(\nu \beta-\operatorname{Tr}_{L / F(\alpha)}(\beta)\right)\right) .
$$

Proof

Let $\varepsilon=a \alpha+b\left(\nu \beta-\operatorname{Tr}_{L / F(\alpha)}(\beta)\right) \in L$. Then we have

$$
\begin{aligned}
\operatorname{Tr}_{L / F(\alpha)}(\varepsilon)= & a \alpha \operatorname{Tr}_{L / F(\alpha)}(1)+b \nu \operatorname{Tr}_{L / F(\alpha)}(\beta) \\
& -b \operatorname{Tr}_{L / F(\alpha)}(\beta) \operatorname{Tr}_{L / F(\alpha)}(1) \\
= & a \alpha \nu .
\end{aligned}
$$

Since $L$ is an abelian extension of $F$, so is $F(\varepsilon)$ by Galois theory. Thus, $\varepsilon^{\sigma} \in F(\varepsilon)$ for any $\sigma \in \operatorname{Gal}(L / F)$, and hence, $\operatorname{Tr}_{L / F(\alpha)}(\varepsilon) \in F(\varepsilon)$. This implies that $\alpha \in F(\varepsilon)$ 
by (6.2). Therefore, we obtain

$$
F(\varepsilon)=F(\alpha, \varepsilon)=F\left(\alpha, \varepsilon-a \alpha+b \operatorname{Tr}_{L / F(\alpha)}(\beta)\right)=F(\alpha, b \nu \beta)=L .
$$

\section{THEOREM 6.4}

Let $\ell$ and $p$ be odd primes, let $n=(\ell-1) / 2$, and let $\mu \in \mathbb{Z}_{>0}$. Let $\mathbf{r}, \mathbf{s}, z_{\ell}$, and $A_{\ell}(\mathbf{r}, \mathbf{s})$ be as above. Assume that $z_{\ell}$ is neither a zero nor a pole of $\prod_{i=1}^{n+1} \Phi_{[\mathbf{r}, \mathbf{s} ; \mu, i]}(z)_{p}$. If $p \nmid \ell h_{\ell}^{+} n \cdot \operatorname{det}\left(A_{\ell}(\mathbf{r}, \mathbf{s})\right)$, then we have

$$
K_{2 \mu-1-\alpha}=k_{2 \mu-1-\alpha}\left(\sum_{i=1}^{n+1} \Phi_{[\mathbf{r}, \mathbf{s} ; \mu, i]}\left(z_{\ell}\right)_{p}^{p^{\alpha}}\right)
$$

for $\alpha=0,1, \ldots, \mu-1$. Moreover, if $\operatorname{dim}_{\mathbb{Z} / p \mathbb{Z}}\left(H_{1} / S_{2}\right)=n-1$, then we have

$$
k_{2 \mu-\alpha}=k_{\mu}\left(\sum_{i=1}^{n+1} \Phi_{[\mathbf{r}, \mathbf{s} ; \mu, i]}\left(z_{\ell}\right)_{p}^{p^{\alpha}}\right)
$$

for $\alpha=0,1, \ldots, \mu-1$.

\section{Proof}

Let $\mathbf{x}_{\mu, i}=r_{i} / p^{\mu}$ and $\mathbf{y}_{\mu, i}=s_{i} / p^{\mu}$ for each $\mu$ and $i$. Then $\mathbf{x}_{\mu, i}=\mathbf{x}_{1, i} / p^{\mu-1}$ and $\mathbf{y}_{\mu, i}=\mathbf{y}_{1, i} / p^{\mu-1}$. By Lemma $6.1, \Phi_{[\mathbf{r}, \mathbf{s} ; \mu, i]}\left(z_{\ell}\right)_{p}^{p^{\alpha}} \in K_{2 \mu-1-\alpha}$. It then follows from (6.1) that for $1 \leq i, j \leq n+1$

$$
\begin{aligned}
& \left(\Phi_{[\mathbf{r}, \mathbf{s} ; \mu, i]}\left(z_{\ell}\right)_{p}^{p^{\alpha}}\right){ }^{\left(\frac{K_{2 \mu-1-\alpha} / k}{\left(\omega_{2 \mu-1-\alpha, j}\right)}\right)} \\
& \quad=e\left(-p^{2 \mu-1} \cdot{ }^{t} \mathbf{x}_{\mu, i} \mathbf{y}_{\mu, i}\right) e\left(\frac{{ }^{t} \mathbf{x}_{\mu, i} b_{2 \mu-1, j}-{ }^{t} a_{2 \mu-1, j} \mathbf{y}_{\mu, i}}{2}\right) \Phi_{[\mathbf{r}, \mathbf{s} ; \mu, i]}\left(z_{\ell}\right)_{p}^{p^{\alpha}},
\end{aligned}
$$

where $a_{2 \mu-1, j}, b_{2 \mu-1, j} \in 2 p^{\mu-1} \mathbb{Z}^{n}$ such that

$$
\begin{aligned}
{\left[\begin{array}{l}
a_{2 \mu-1, j} \\
b_{2 \mu-1, j}
\end{array}\right] } & =2 p^{2 \mu-1} \cdot{ }^{t} h\left(\varphi^{+}\left(\zeta^{j}\right)\right)\left[\begin{array}{l}
\mathbf{x}_{\mu, i} \\
\mathbf{y}_{\mu, i}
\end{array}\right] \\
& =2 p^{\mu} \cdot{ }^{t} h\left(\varphi^{+}\left(\zeta^{j}\right)\right)\left[\begin{array}{l}
\mathbf{x}_{1, i} \\
\mathbf{y}_{1, i}
\end{array}\right] \\
& =p^{\mu-1}\left[\begin{array}{l}
a_{1, j} \\
b_{1, j}
\end{array}\right] .
\end{aligned}
$$

We ensure that

$$
\begin{aligned}
& \left(\Phi_{[\mathbf{r}, \mathbf{s} ; \mu, i]}\left(z_{\ell}\right)_{p}^{p^{\alpha}}\right)\left(\frac{K_{2 \mu-1-\alpha} / k}{\left(\omega_{2 \mu-1-\alpha, j}\right)}\right) \\
& \quad=e\left(-p \cdot{ }^{t} \mathbf{x}_{1, i} \mathbf{y}_{1, i}\right) e\left(\frac{{ }^{t} \mathbf{x}_{1, i} b_{1, j}-{ }^{t} a_{1, j} \mathbf{y}_{1, i}}{2}\right) \Phi_{[\mathbf{r}, \mathbf{s} ; \mu, i]}\left(z_{\ell}\right)_{p}^{p^{\alpha}} \\
& \quad=\zeta_{p}^{c_{i j}} \Phi_{[\mathbf{r}, \mathbf{s} ; \mu, i]}\left(z_{\ell}\right)_{p}^{p^{\alpha}},
\end{aligned}
$$

where $c_{i j}$ is the $(i, j)$ th entry of $A_{\ell}(\mathbf{r}, \mathbf{s})$. Here, we observe that for $1 \leq i \leq n+$ 1 there exists $\gamma_{i} \in k_{2 \mu-1-\alpha}\left(\Phi_{[\mathbf{r}, \mathbf{s} ; \mu, 1]}\left(z_{\ell}\right)_{p}^{p^{\alpha}}, \Phi_{[\mathbf{r}, \mathbf{s} ; \mu, 2]}\left(z_{\ell}\right)_{p}^{p^{\alpha}}, \ldots, \Phi_{[\mathbf{r}, \mathbf{s} ; \mu, n+1]}\left(z_{\ell}\right)_{p}^{p^{\alpha}}\right)$ 
with

$$
\gamma_{i}^{\left(\frac{K_{2 \mu-1-\alpha} / k}{\left(\omega_{2 \mu-1-\alpha, j}\right)}\right)}= \begin{cases}\zeta_{p} \gamma_{i} & \text { if } j=i, \\ \gamma_{i} & \text { if } j \neq i,\end{cases}
$$

because $p \nmid \operatorname{det}\left(A_{\ell}(\mathbf{r}, \mathbf{s})\right)$. Since $\left|\operatorname{Gal}\left(K_{2 \mu-1-\alpha} / k_{2 \mu-1-\alpha}\right)\right| \leq p^{n+1}$ by (4.1), we deduce

(6.6)

$$
\begin{aligned}
K_{2 \mu-1-\alpha} & =k_{2 \mu-1-\alpha}\left(\gamma_{1}, \gamma_{2}, \ldots, \gamma_{n+1}\right) \\
& =k_{2 \mu-1-\alpha}\left(\Phi_{[\mathbf{r}, \mathbf{s} ; \mu, 1]}\left(z_{\ell}\right)_{p}^{p^{\alpha}}, \Phi_{[\mathbf{r}, \mathbf{s} ; \mu, 2]}\left(z_{\ell}\right)_{p}^{p^{\alpha}}, \ldots, \Phi_{[\mathbf{r}, \mathbf{s} ; \mu, n+1]}\left(z_{\ell}\right)_{p}^{p^{\alpha}}\right) .
\end{aligned}
$$

Now, let $L_{i}=k_{2 \mu-1-\alpha}\left(\Phi_{[\mathbf{r}, \mathbf{s} ; \mu, 1]}\left(z_{\ell}\right)_{p}^{p^{\alpha}}, \Phi_{[\mathbf{r}, \mathbf{s} ; \mu, 2]}\left(z_{\ell}\right)_{p}^{p^{\alpha}}, \ldots, \Phi_{[\mathbf{r}, \mathbf{s} ; \mu, i]}\left(z_{\ell}\right)_{p}^{p^{\alpha}}\right)$ for each $1 \leq i \leq n+1$. Suppose that $L_{m}=k_{2 \mu-1-\alpha}\left(\sum_{i=1}^{m} \Phi_{[\mathbf{r}, \mathbf{s} ; \mu, i]}\left(z_{\ell}\right)_{p}^{p^{\alpha}}\right)$ for some integer $1 \leq m \leq n$. Note that $\left[L_{m+1}: L_{m}\right]=p$ and

$$
\operatorname{Tr}_{L_{m+1} / L_{m}}\left(\Phi_{[\mathbf{r}, \mathbf{s} ; \mu, m+1]}\left(z_{\ell}\right)_{p}^{p^{\alpha}}\right)=\sum_{j=0}^{p-1} \zeta_{p}^{j} \Phi_{[\mathbf{r}, \mathbf{s} ; \mu, m+1]}\left(z_{\ell}\right)_{p}^{p^{\alpha}}=0,
$$

due to the fact that $\sum_{j=0}^{p-1} \zeta_{p}^{j}=0$. Hence, if we take $a=1$ and $b=1 / p$, then by Lemma 6.3 we obtain

$$
\begin{aligned}
L_{m+1} & =k_{2 \mu-1-\alpha}\left(\sum_{i=1}^{m} \Phi_{[\mathbf{r}, \mathbf{s} ; \mu, i]}\left(z_{\ell}\right)_{p}^{p^{\alpha}}, \Phi_{[\mathbf{r}, \mathbf{s} ; \mu, m+1]}\left(z_{\ell}\right)_{p}^{p^{\alpha}}\right) \\
& =k_{2 \mu-1-\alpha}\left(\sum_{i=1}^{m+1} \Phi_{[\mathbf{r}, \mathbf{s} ; \mu, i]}\left(z_{\ell}\right)_{p}^{p^{\alpha}}\right) .
\end{aligned}
$$

Therefore, (6.3) is proved by induction and (6.6).

If $\operatorname{dim}_{\mathbb{Z} / p \mathbb{Z}}\left(H_{1} / S_{2}\right)=n-1$, then by the proof of Corollary 4.6 we get

$$
\left|\operatorname{Gal}\left(k_{2 \mu-\alpha} / k_{2 \mu-1-\alpha}\right)\right|=p^{n+1} .
$$

Since $\left|\operatorname{Gal}\left(K_{2 \mu-1-\alpha} / k_{2 \mu-1-\alpha}\right)\right|=p^{n+1}$ and $K_{2 \mu-1-\alpha} \subset k_{2 \mu-\alpha}$, we conclude that $K_{2 \mu-1-\alpha}=k_{2 \mu-\alpha}$. Hence, by (6.6)

$$
\begin{aligned}
k_{2 \mu-\alpha} & =k_{2 \mu-1-\alpha}\left(\Phi_{[\mathbf{r}, \mathbf{s} ; \mu, 1]}\left(z_{\ell}\right)_{p}^{p^{\alpha}}, \Phi_{[\mathbf{r}, \mathbf{s} ; \mu, 2]}\left(z_{\ell}\right)_{p}^{p^{\alpha}}, \ldots, \Phi_{[\mathbf{r}, \mathbf{s} ; \mu, n+1]}\left(z_{\ell}\right)_{p}^{p^{\alpha}}\right) \\
& =k_{\mu}\left(\Phi_{[\mathbf{r}, \mathbf{s} ; \mu, 1]}\left(z_{\ell}\right)_{p}^{p^{\alpha}}, \Phi_{[\mathbf{r}, \mathbf{s} ; \mu, 2]}\left(z_{\ell}\right)_{p}^{p^{\alpha}}, \ldots, \Phi_{[\mathbf{r}, \mathbf{s} ; \mu, n+1]}\left(z_{\ell}\right)_{p}^{p^{\alpha}}\right)
\end{aligned}
$$

for $\quad \alpha=0,1, \ldots, \mu-1$. Let $L_{i}^{\prime}=k_{\mu}\left(\Phi_{[\mathbf{r}, \mathbf{s} ; \mu, 1]}\left(z_{\ell}\right)_{p}^{p^{\alpha}}, \Phi_{[\mathbf{r}, \mathbf{s} ; \mu, 2]}\left(z_{\ell}\right)_{p}^{p^{\alpha}}, \ldots\right.$, $\left.\Phi_{[\mathbf{r}, \mathbf{s} ; \mu, i]}\left(z_{\ell}\right)_{p}^{p^{\alpha}}\right)$ for each $1 \leq i \leq n+1$. Suppose that $L_{m}^{\prime}=k_{\mu}\left(\sum_{i=1}^{m} \Phi_{[\mathbf{r}, \mathbf{s} ; \mu, i]}\left(z_{\ell}\right)_{p}^{p^{\alpha}}\right)$ for some integer $1 \leq m \leq n$. Then we have

$$
\begin{aligned}
\operatorname{Tr}_{L_{m+1} / L_{m}^{\prime}}\left(\Phi_{[\mathbf{r}, \mathbf{s} ; \mu, m+1]}\left(z_{\ell}\right)_{p}^{p^{\alpha}}\right) & =\operatorname{Tr}_{L_{m} / L_{m}^{\prime}}\left(\operatorname{Tr}_{L_{m+1} / L_{m}}\left(\Phi_{[\mathbf{r}, \mathbf{s} ; \mu, m+1]}\left(z_{\ell}\right)_{p}^{p^{\alpha}}\right)\right)(=0) \\
& =\operatorname{Tr}_{L_{m+1}^{\prime} / L_{m}^{\prime}}\left(\operatorname{Tr}_{L_{m+1} / L_{m+1}^{\prime}}\left(\Phi_{[\mathbf{r}, \mathbf{s} ; \mu, m+1]}\left(z_{\ell}\right)_{p}^{p^{\alpha}}\right)\right) \\
& =\left[L_{m+1}: L_{m+1}^{\prime}\right] \cdot \operatorname{Tr}_{L_{m+1}^{\prime} / L_{m}^{\prime}}\left(\Phi_{[\mathbf{r}, \mathbf{s} ; \mu, m+1]}\left(z_{\ell}\right)_{p}^{p^{\alpha}}\right)
\end{aligned}
$$


and so $\operatorname{Tr}_{L_{m+1}^{\prime} / L_{m}^{\prime}}\left(\Phi_{[\mathbf{r}, \mathbf{s} ; \mu, m+1]}\left(z_{\ell}\right)_{p}^{p^{\alpha}}\right)=0$. Therefore, by Lemma 6.3

$$
\begin{aligned}
L_{m+1}^{\prime} & =k_{\mu}\left(\sum_{i=1}^{m} \Phi_{[\mathbf{r}, \mathbf{s} ; \mu, i]}\left(z_{\ell}\right)_{p}^{p^{\alpha}}, \Phi_{[\mathbf{r}, \mathbf{s} ; \mu, m+1]}\left(z_{\ell}\right)_{p}^{p^{\alpha}}\right) \\
& =k_{\mu}\left(\sum_{i=1}^{m+1} \Phi_{[\mathbf{r}, \mathbf{s} ; \mu, i]}\left(z_{\ell}\right)_{p}^{p^{\alpha}}\right)
\end{aligned}
$$

and (6.4) is proved again by induction.

Although we omit in the above theorem the case where $p \operatorname{divides} \operatorname{det}\left(A_{\ell}(\mathbf{r}, \mathbf{s})\right)$, by utilizing Theorem 4.5 we might find suitable generators of $K_{\mu}$ over $k_{\mu}$ for each $\mu \in \mathbb{Z}_{>0}$.

Let $\mathbf{r}_{0}=\left[r_{i}\right]_{1 \leq i \leq n+1}$ and $\mathbf{s}_{0}=\left[s_{i}\right]_{1 \leq i \leq n+1}$, where $r_{i}={ }^{t}\left[\begin{array}{llll}1 & 0 & \cdots & 0\end{array}\right] \in \mathbb{Z}^{n}$ and $s_{i}={ }^{t}\left[\left(s_{i}\right)_{j}\right]_{1 \leq j \leq n} \in \mathbb{Z}^{n}$ for $1 \leq i \leq n+1$ with

$$
\left(s_{i}\right)_{j}= \begin{cases}1 & \text { if } j<i, \\ 0 & \text { otherwise. }\end{cases}
$$

Here we observe that $\Phi_{\left[\mathbf{r}_{0}, \mathbf{s}_{0} ; \mu, i\right]}(z)_{p}$ is not identically zero for all $\mu$ and $i$ by Proposition 5.3.

\section{COROLLARY 6.5}

Let $\ell$ and $p$ be odd primes, and let $z_{\ell}$ be as above. Put $n=(\ell-1) / 2$, and let $\mu \in \mathbb{Z}_{>0}$. Further, we assume that $z_{\ell}$ is not a zero of $\prod_{i=1}^{n+1} \Phi_{\left[\mathbf{r}_{0}, \mathbf{s}_{0} ; \mu, i\right]}(z)_{p}$.

(i) Let $\ell=7$. If $p \neq 3,7$, then we have

$$
K_{2 \mu-1-\alpha}=k_{2 \mu-1-\alpha}\left(\sum_{i=1}^{4} \Phi_{\left[\mathbf{r}_{0}, \mathbf{s}_{0} ; \mu, i\right]}\left(z_{7}\right)_{p}^{p^{\alpha}}\right)
$$

for $0 \leq \alpha \leq \mu-1$.

(ii) Let $\ell=11$. If $p \neq 3,5,11$, then we have

$$
K_{2 \mu-1-\alpha}=k_{2 \mu-1-\alpha}\left(\sum_{i=1}^{6} \Phi_{\left[\mathbf{r}_{0}, \mathbf{s}_{0} ; \mu, i\right]}\left(z_{11}\right)_{p}^{p^{\alpha}}\right)
$$

for $0 \leq \alpha \leq \mu-1$. If $p=3$, then we get

$$
K_{2 \mu-1-\alpha}=k_{2 \mu-1-\alpha}\left(\sum_{i=1}^{5} \Phi_{\left[\mathbf{r}_{0}, \mathbf{s}_{0} ; \mu, i\right]}\left(z_{11}\right)_{3}^{3^{\alpha}}\right) .
$$

(iii) Let $\ell=13$. If $p \neq 3,5,13$, then we have

$$
K_{2 \mu-1-\alpha}=k_{2 \mu-1-\alpha}\left(\sum_{i=1}^{7} \Phi_{\left[\mathbf{r}_{0}, \mathbf{s}_{0} ; \mu, i\right]}\left(z_{13}\right)_{p}^{p^{\alpha}}\right)
$$

for $0 \leq \alpha \leq \mu-1$. If $p=5$, then we obtain

$$
K_{2 \mu-1-\alpha}=k_{2 \mu-1-\alpha}\left(\sum_{i=1}^{6} \Phi_{\left[\mathbf{r}_{0}, \mathbf{s}_{0} ; \mu, i\right]}\left(z_{13}\right)_{5}^{5^{\alpha}}\right) .
$$


(iv) Let $\ell=5$. Then we have

$$
K_{2 \mu-1-\alpha}=k_{2 \mu-1-\alpha}\left(\zeta_{p^{2 \mu-\alpha}}+\Phi_{\left[\mathbf{r}_{0}, \mathbf{s}_{0} ; \mu, 1\right]}\left(z_{5}\right)_{p}^{p^{\alpha}}+\Phi_{\left[\mathbf{r}_{0}, \mathbf{s}_{0} ; \mu, 3\right]}\left(z_{5}\right)_{p}^{p^{\alpha}}\right)
$$

for $0 \leq \alpha \leq \mu-1$.

\section{Proof}

Let the matrix $M_{\ell}(p)$ be as in Lemma 4.4. Note that $h_{\ell}^{+}=1$ for $\ell \leq 67$ (see [30, p. 352]). We can show that $z_{\ell}$ is not a pole of $\prod_{i=1}^{n+1} \Phi_{\left[\mathbf{r}_{0}, \mathbf{s}_{0} ; \mu, i\right]}(z)_{p}$ for $\ell \leq 17$ by utilizing the RiemannTheta command in Maple. lows:

(i) Using (6.1) we can find $\Phi_{\left[\mathbf{r}_{0}, \mathbf{s}_{0} ; 1, i\right]}\left(z_{7}\right)_{p}^{\left(\frac{K_{1} / k}{\left(\omega_{1, j}\right)}\right)}$ for each $1 \leq i, j \leq 4$ as fol-

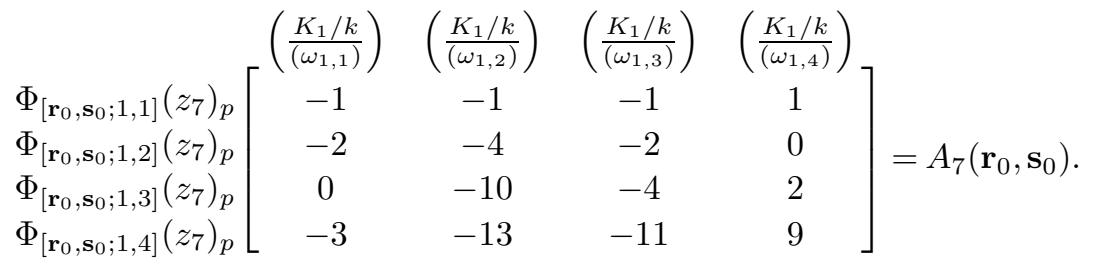

Since $\operatorname{det}\left(A_{7}\left(\mathbf{r}_{0}, \mathbf{s}_{0}\right)\right)=2^{6}$ is prime to $p,(6.7)$ is true by Theorem 6.4.

(ii) First, suppose that $p \neq 3,5,11$. In a similar way as in (i) we obtain

$$
A_{11}\left(\mathbf{r}_{0}, \mathbf{s}_{0}\right)=\left[\begin{array}{cccccc}
-1 & -1 & -1 & -1 & -1 & 1 \\
-2 & -4 & -2 & -4 & -2 & 0 \\
-4 & -6 & -4 & -10 & 0 & -2 \\
-7 & -3 & -11 & -21 & -3 & 1 \\
-7 & -5 & -25 & -29 & -1 & -1 \\
-10 & -2 & -48 & -34 & -6 & 4
\end{array}\right]
$$

Since $\operatorname{det}\left(A_{11}\left(\mathbf{r}_{0}, \mathbf{s}_{0}\right)\right)=2^{7} \cdot 3 \cdot 5^{2}$ is prime to $p$, we get (6.8) by Theorem 6.4. If $p=3$, then the rank of $M_{11}(3)$ is equal to 5 . Since $p \nmid 11 \cdot 5$, by Theorem 4.5 we deduce $\operatorname{Gal}\left(K_{\mu} / k_{\mu}\right) \cong(\mathbb{Z} / 3 \mathbb{Z})^{5}$ for all $\mu \in \mathbb{Z}_{>0}$. We observe that the determinant of the matrix

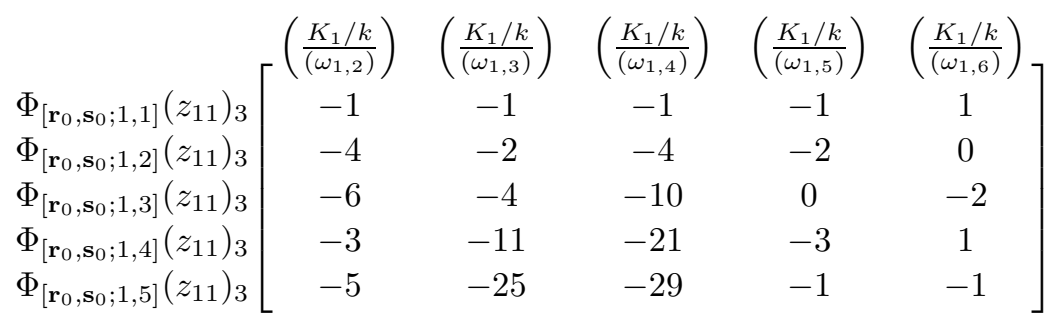

is equal to $2^{5} \cdot 5 \cdot 11$, which is prime to 3 . Using Lemma 6.3 and (6.5) we can conclude (6.9). 
(iii) First, suppose that $p \neq 3,5,13$. Then we derive

$$
A_{13}\left(\mathbf{r}_{0}, \mathbf{s}_{0}\right)=\left[\begin{array}{ccccccc}
-1 & -1 & -1 & -1 & -1 & -1 & 1 \\
-2 & -4 & -2 & -4 & -2 & -4 & 2 \\
0 & -10 & -4 & -6 & -4 & -10 & 8 \\
5 & -19 & -7 & -11 & -11 & -13 & 11 \\
7 & -35 & -13 & -13 & -19 & -15 & 13 \\
2 & -60 & -18 & -8 & -32 & -22 & 20 \\
-10 & -84 & -28 & 2 & -54 & -22 & 20
\end{array}\right] .
$$

Since $\operatorname{det}\left(A_{13}\left(\mathbf{r}_{0}, \mathbf{s}_{0}\right)\right)=-2^{12} \cdot 5^{2}$ is prime to $p$, we have (6.10) again by Theorem 6.4. If $p=5$, then the rank of $M_{13}(5)$ is equal to 6 . Since $p \nmid 13 \cdot 6$, it follows from Theorem 4.5 that $\operatorname{Gal}\left(K_{\mu} / k_{\mu}\right) \cong(\mathbb{Z} / 5 \mathbb{Z})^{6}$ for all $\mu \in \mathbb{Z}_{>0}$. Observe that the determinant of the matrix

\begin{tabular}{|c|c|c|c|c|c|c|}
\hline & $\left(\frac{K_{1} / k}{\left(\omega_{1,2}\right)}\right)$ & $\left(\frac{K_{1} / k}{\left(\omega_{1,3}\right)}\right)$ & $\left(\frac{K_{1} / k}{\left(\omega_{1,4}\right)}\right)$ & $\left(\frac{K_{1} / k}{\left(\omega_{1,5}\right)}\right)$ & $\left(\frac{K_{1} / k}{\left(\omega_{1,6}\right)}\right)$ & $\left(\frac{K_{1} / k}{\left(\omega_{1,7}\right)}\right)$ \\
\hline$\Phi_{\left[\mathbf{r}_{0}, \mathbf{s}_{0} ; 1,1\right]}\left(z_{13}\right)_{5}$ & -1 & -1 & -1 & -1 & -1 & 1 \\
\hline$\Phi_{\left[\mathbf{r}_{0}, \mathbf{s}_{0} ; 1,2\right]}\left(z_{13}\right)_{5}$ & -4 & -2 & -4 & -2 & -4 & 2 \\
\hline$\Phi_{\left[\mathbf{r}_{0}, \mathbf{s}_{0} ; 1,3\right]}\left(z_{13}\right)_{5}$ & -10 & -4 & -6 & -4 & -10 & 8 \\
\hline$\Phi_{\left[\mathbf{r}_{0}, \mathbf{s}_{0} ; 1,4\right]}\left(z_{13}\right)_{5}$ & -19 & -7 & -11 & -11 & -13 & 11 \\
\hline$\Phi_{\left[\mathbf{r}_{0}, \mathbf{s}_{0} ; 1,5\right]}\left(z_{13}\right)_{5}$ & -35 & -13 & -13 & -19 & -15 & 13 \\
\hline$\Phi_{\left[\mathbf{r}_{0}, \mathbf{s}_{0}: 1,6\right]}\left(z_{13}\right)_{5}$ & -60 & -18 & -8 & -32 & -22 & 20 \\
\hline
\end{tabular}

is equal to $-2^{7} \cdot 31$, which is prime to 5 . Using Lemma 6.3 and (6.5) we can deduce (6.11).

(iv) In this case, $\operatorname{det}\left(A_{5}\left(\mathbf{r}_{0}, \mathbf{s}_{0}\right)\right)=0$ so we should find another generator of $K_{2 \mu-1-\alpha}$ over $k_{2 \mu-1-\alpha}$. By [14, p. 316], $H_{2 \mu-1-\alpha} / S_{2 \mu-\alpha}$ is generated by real units of $k$ for any odd prime $p$. Using the idea in the proof of Theorem 4.5, one can show that $\left(\varphi^{*}\left(S_{2 \mu-1-\alpha}\right) \cap H_{2 \mu-1-\alpha}\right) / S_{2 \mu-\alpha}=\{0\}$, and so $\Phi_{\left[\mathbf{r}_{0}, \mathbf{s}_{0} ; \mu, i\right]}\left(z_{5}\right)_{p}^{p^{\alpha}} \in$ $K_{2 \mu-1-\alpha}$ for $1 \leq i \leq 3$. Note that $\zeta_{p^{2 \mu-\alpha}} \in \mathcal{F}_{2 p^{2 \mu-\alpha}}$ is $R_{2 p^{2 \mu-\alpha}}$-invariant; hence, $\zeta_{p^{2 \mu-\alpha}} \in K_{2 \mu-1-\alpha}$ by Proposition 3.2. Since $N_{k / \mathbb{Q}}\left(\omega_{2 \mu-1-\alpha, j}\right) \equiv 1-2 p^{2 \mu-1-\alpha}$ $\left(\bmod 2 p^{2 \mu-\alpha}\right)$ for $1 \leq j \leq 3$, we get

$$
\zeta_{p^{2 \mu-\alpha}}^{\left(\frac{K_{2 \mu-1-\alpha} / k}{\left(\omega_{2 \mu-1-\alpha, j}\right)}\right.}=\zeta_{p}^{-2} \zeta_{p^{2 \mu-\alpha}} \quad \text { for } 1 \leq j \leq 3
$$

Now, observe that the determinant of the matrix

$$
\oint_{\Phi_{\left[\mathbf{r}_{0}, \mathbf{s}_{0} ; 1,1\right]}\left(z_{5}\right)_{p}}\left[\begin{array}{ccc}
\left(\frac{K_{1} / k}{\left(\omega_{1,1}\right)}\right) & \left(\frac{K_{1} / k}{\left(\omega_{1,2}\right)}\right) & \left(\frac{K_{1} / k}{\left(\omega_{1,3}\right)}\right) \\
-2 & -2 & -2 \\
-1 & -1 & 1 \\
-4 & -6 & 4
\end{array}\right]
$$

is equal to $-2^{3}$, which is prime to $p$. Therefore, we obtain (6.12) by Lemma 6.3, (6.5), and (6.13).

\section{REMARK 6.6}

(i) Especially when $\mu=1$, Corollary 6.5(iv) is reduced to Komatsu's work (see [14, Proposition 1]) with a few different ingredients. 
Table 2

\begin{tabular}{|c|c|}
\hline$\ell$ & prime factors of $\operatorname{det}\left(A_{\ell}\left(\mathbf{r}_{0}, \mathbf{s}_{0}\right)\right)$ \\
\hline 3 & 2 \\
\hline 5 & 0 \\
\hline 7 & 2 \\
\hline 11 & $2,3,5$ \\
\hline 13 & 2,5 \\
\hline 17 & $2,7,17,43$ \\
\hline 19 & $2,3,36137$ \\
\hline 23 & $2,3,11,13,29,89,241$ \\
\hline 29 & $2,3,5,13,113,58057291$ \\
\hline 31 & $2,3,31,109621,1216387$ \\
\hline 37 & $2,5,13,37,53,109,10138325056259$ \\
\hline 41 & $2,5,11,17,41,439,1667,166013,203381$ \\
\hline 43 & $2,3,19,43,211,281345721890371109$ \\
\hline 47 & $2,5,83,139,5323,178481,6167669171116393$ \\
\hline 53 & $2,3,5,139,157,1613,4889,1579367,28153859844430949$ \\
\hline 59 & $2,3,59,233,3033169,1899468180409634452730252070517$ \\
\hline 61 & $2,5,11,13,41,1321,1861,1142941857599125232990619467569$ \\
\hline 67 & $2,3,67,683,12739,20857,513881,1858283767,986862333655510350967$ \\
\hline 71 & $\begin{array}{l}2, \quad 5, \quad 7, \quad 31, \quad 79, \quad 127, \quad 1129, \quad 79241, \quad 122921, \\
3087543529906501\end{array}$ \\
\hline 73 & $2,7,73,79,89,16747,134353,5754557119657,1150806776867233,1190899$ \\
\hline 79 & $\begin{array}{l}2,5,7,13,29,53,1427,3847,8191,121369,377911,1842497,51176893, \\
357204083,32170088152177\end{array}$ \\
\hline 83 & $\begin{array}{l}2, \quad 3, \quad 13, \quad 17387, \quad 279405653, \quad 43059261982072584626787705301351, \\
8831418697,758583423553\end{array}$ \\
\hline 89 & $\begin{array}{l}2,17,23,89,113,313629821584641896139082338756559409, \\
118401449,22482210593\end{array}$ \\
\hline
\end{tabular}

(ii) Table 2 gives the prime factors of $\operatorname{det}\left(A_{\ell}\left(\mathbf{r}_{0}, \mathbf{s}_{0}\right)\right)$ for $\ell \leq 89$.

\section{References}

[1] T. M. Apostol, Introduction to Analytic Number Theory, Undergrad. Texts Math., Springer, New York, 1976. MR 0434929.

[2] B. Cais and B. Conrad, Modular curves and Ramanujan's continued fraction, J. Reine Angew. Math. 597 (2006), 27-104. MR 2264315.

DOI 10.1515/CRELLE.2006.063.

[3] I. Chen and N. Yui, "Singular values of Thompson series" in Groups, Difference Sets, and the Monster (Columbus, OH, 1993), Ohio State Univ. Math. Res. Inst. Publ. 4, de Gruyter, Berlin, 1996, 255-326. MR 1400423. 
[4] B. Cho and J. K. Koo, Construction of class fields over imaginary quadratic fields and applications, Q. J. Math. 61 (2010), 199-216. MR 2646085. DOI 10.1093/qmath/han035.

[5] J. Cougnard, Conditions nécessaires de monogénéité: Application aux extensions cycliques de degré premier $\ell \geq 5$ d'un corps quadratique imaginaire, J. Lond. Math. Soc. (2) 37 (1988), 73-87. MR 0921746. DOI 10.1112/jlms/s2-37.121.73.

[6] J. Cougnard and M. Vérant, Monogénéité de l'anneau des entiers de corps de classes de rayon de corps quadratiques, Sém. Théor. Nombres Bordeaux (2) 4 (1992), 53-74. MR 1183918.

[7] D. A. Cox, Primes of the Form $x^{2}+n y^{2}$ : Fermat, Class Field Theory and Complex Multiplication, Wiley, New York, 1989. MR 1028322.

[8] D. A. Cox, J. McKay, and P. Stevenhagen, Principal moduli and class fields, Bull. Lond. Math. Soc. 36 (2004), 3-12. MR 2011972.

DOI 10.1112/S0024609303002583.

[9] M. Eichler, Der Hilbertsche Klassenkörper eines imaginärquadratischen Zahlkörpers, Math. Z. 64 (1956), 229-242. MR 0086096.

[10] H. Hasse, Neue Bergründung der komplexen Multiplikation, I: Einordnung in die allgemeine Klassenkörpertheorie, J. Reine Angew. Math. 157 (1927), 115-140; II: Aufbau ohne Benutzung der allgemeinen Klassenkörpertheorie, 165 (1931), 64-88. MR 1581113; MR 1581274. DOI 10.1515/crll.1931.165.64.

[11] J. Igusa, On the graded ring of theta-constants, II, Amer. J. Math. 88 (1966), 221-236. MR 0200482.

[12] H. Y. Jung, J. K. Koo, and D. H. Shin, Normal bases of ray class fields over imaginary quadratic fields, Math. Z. 271 (2012), 109-116. MR 2917135. DOI 10.1007/s00209-011-0854-2.

[13] H. Klingen, Introductory Lectures on Siegel Modular Forms, Cambridge Stud. Adv. Math. 20, Cambridge Univ. Press, Cambridge, 1990. MR 1046630. DOI 10.1017/CBO9780511619878.

[14] K. Komatsu, Construction of a normal basis by special values of Siegel modular functions, Proc. Amer. Math. Soc. 128 (2000), 315-323. MR 1707153. DOI 10.1090/S0002-9939-99-05601-4.

[15] S. Lang, Elliptic Functions, with an appendix by J. Tate, 2nd ed., Grad. Texts in Math. 112, Springer, New York, 1987. MR 0890960.

DOI 10.1007/978-1-4612-4752-4.

[16] J. J. Liang, On the integral basis of the maximal real subfield of a cyclotomic field, J. Reine Angew. Math. 286/287 (1976), 223-226. MR 0419402.

[17] B. Mazur, How can we construct abelian Galois extensions of basic number fields?, Bull. Amer. Math. Soc. (N.S.) 48 (2011), 155-209. MR 2774089. DOI 10.1090/S0273-0979-2011-01326-X.

[18] K. Ramachandra, Some applications of Kronecker's limit formulas, Ann. of Math. (2) 80 (1964), 104-148. MR 0164950. 
[19] K. A. Ribet, A modular construction of unramified p-extensions of $\mathbb{Q}\left(\mu_{p}\right)$, Invent. Math. 34 (1976), 151-162. MR 0419403.

[20] R. Schertz, L-Reihen in imaginär-quadratischen Zahlkörpern und ihre Anwendung auf Klassenzahlprobleme bei quadratischen und biquadratischen Zahlkörpern, I, J. Reine Angew. Math. 262/263 (1973), 120-133. MR 0332731.

[21] - Weber's class invariants revisited, J. Théor. Nombres Bordeaux 14 (2002), 325-343. MR 1926005.

[22] Complex Multiplication, New Math. Monogr. 15, Cambridge Univ. Press, Cambridge, 2010. MR 2641876. DOI 10.1017/CBO9780511776892.

[23] G. Shimura, On the class-fields obtained by complex multiplication of abelian varieties, Osaka J. Math. 14 (1962), 33-44. MR 0170893.

[24] On canonical models of arithmetic quotients of bounded symmetric domains, Ann. of Math. (2) 91 (1970), 144-222. MR 0257031.

[25] - On canonical models of arithmetic quotients of bounded symmetric domains, II, Ann. of Math. (2) 92 (1970), 528-549. MR 0292758.

[26] , Theta functions with complex multiplication, Duke Math. J. 43 (1976), 673-696. MR 0424705.

[27] Abelian Varieties with Complex Multiplication and Modular Functions, Princeton Math. Ser. 46, Princeton Univ. Press, Princeton, 1998. MR 1492449.

[28] P. Stevenhagen, "Hilbert's 12th problem, complex multiplication and Shimura reciprocity" in Class Field Theory-Its Centenary and Prospect (Tokyo, 1998), Adv. Stud. Pure Math. 30, Math. Soc. Japan, Tokyo, 2001, 161-176. MR 1846457.

[29] T. Takagi, Über eine Theorie des relativ-Abelschen Zahlkörpers, J. Coll. Sci. Univ. Tokyo 41 (1920), 1-133.

[30] L. C. Washington, Introduction to Cyclotomic Fields, Grad. Texts in Math. 83, Springer, New York, 1982. MR 0718674. DOI 10.1007/978-1-4684-0133-2.

Koo: Department of Mathematical Sciences, KAIST, Daejeon, Republic of Korea; jkkoo@math.kaist.ac.kr

Yoon: Department of Mathematical Sciences, KAIST, Daejeon, Republic of Korea; math_dsyoon@kaist.ac.kr 